This item was submitted to Loughborough's Research Repository by the author.

Items in Figshare are protected by copyright, with all rights reserved, unless otherwise indicated.

\title{
An integrated approach to model strain localization bands in magnesium alloys
}

PLEASE CITE THE PUBLISHED VERSION

https://doi.org/10.1007/s00466-017-1480-6

\section{PUBLISHER}

(C) Springer

\section{VERSION}

AM (Accepted Manuscript)

\section{PUBLISHER STATEMENT}

This work is made available according to the conditions of the Creative Commons Attribution-NonCommercialNoDerivatives 4.0 International (CC BY-NC-ND 4.0) licence. Full details of this licence are available at: https://creativecommons.org/licenses/by-nc-nd/4.0/

\section{LICENCE}

CC BY-NC-ND 4.0

\section{REPOSITORY RECORD}

Baxevanakis, Konstantinos, C. Mo, M. Cabal, and A. Kontsos. 2017. "An Integrated Approach to Model Strain Localization Bands in Magnesium Alloys”. Loughborough University. https://hdl.handle.net/2134/26817. 


\author{
An Integrated Approach to Model \\ Strain Localization Bands in Magnesium Alloys \\ K. P. Baxevanakis ${ }^{1,2}, \mathrm{C} . \mathrm{Mo}^{2}, \mathrm{M} . \mathrm{Cabal}^{2}$ and A. Kontsos ${ }^{2}{ }^{*}$ \\ ${ }^{1}$ Wolfson School of Mechanical, Electrical and Manufacturing Engineering, \\ Loughborough University, LE11 3TU, UK \\ ${ }^{2}$ Theoretical $\& 3$ Applied Mechanics Group, Mechanical Engineering 83 Mechanics Department, \\ Drexel University, 3141 Chestnut Street, Philadelphia, PA 19104 \\ *Corresponding author: akontsos@coe.drexel.edu, (215)8952297
}

\begin{abstract}
Strain localization bands (SLBs) that appear at early stages of deformation of magnesium alloys have been recently associated with heterogeneous activation of deformation twinning. Experimental evidence has demonstrated that such "Lüders-type" band formations dominate the overall mechanical behavior of these alloys resulting in sigmoidal type stress-strain curves with a distinct plateau followed by pronounced anisotropic hardening. To evaluate the role of SLB formation on the local and global mechanical behavior of magnesium alloys, an integrated experimental/computational approach is presented. The computational part is developed based on custom subroutines implemented in a Finite Element Method that combine a plasticity model with a stiffness degradation approach. Specific inputs from the characterization and testing measurements to the computational approach are discussed while the numerical results are validated against such available experimental information, confirming the existence of load drops and the intensification of strain accumulation at the time of SLB initiation.
\end{abstract}

Keywords: Strain localization, Magnesium alloys, Twinning, Digital Image Correlation, Finite Element Method 


\section{Introduction}

Several numerical approaches to model strain localization bands (SLBs) and shear bands have been reported in the past, demonstrating the need to describe the role of SLBs in a multitude of materials' phenomena across scales and temperature. In this context, several types of finite element models have been reported. For example, Pietruszczak and Mróz [1] developed a two dimensional model in which a band of prescribed thickness was assumed to pre-exist within the elements when a maximum stress intensity was reached. In addition, Tvergaard et al. [2] analyzed a plane strain tensile test characterized by a phenomenological plasticity model for a nonlinear elastic solid. An alternative approach to enrich the elements-level interpolation with suitably defined shape functions that reproduce localized deformation modes once localization is detected was presented by Ortiz et al. [3]. In a similar study by Belytschko et al. [4], localization zones were embedded in plane strain elements to capture shear localization. Furthermore, Batra and Kim [5] analyzed shear bands emerging in a thermally softening viscoplastic block under simple shear deformation. Later, Ramakrishnan and Atluri [6] modeled shear band formation in plane strain tension and compression tests by introducing a plane of lower shear strength. Furthermore, Shaw and Kyriakides [7] created Lüders band instabilities in shape memory alloy tensile strips by introducing geometric imperfections in the form of small indentations along the specimen's side. In addition, Tsukahara and Iung [8] analyzed Lüders band formation and propagation in a tensile specimen by introducing a local stress-plastic strain law and an evolution rule. Shear bands were also studied in relation to voids [9].

More recently, crystal plasticity type finite element simulations were employed to investigate the effect of texture on the development of strain localization. Specifically, Kuroda and Tvergaard 
[10] developed a Taylor-type polycrystalline model to obtain the critical strain at the onset of shear banding and its corresponding orientation in $\mathrm{Al}$ alloys. Moreover, Jia et al. [11] introduced a noncrystallographic shear band mechanism in addition to dislocation and twinning in a crystal plasticity finite element code, and investigated the crystal orientation effect on the shear band development in $\alpha$-Brass single crystal [12].

Physically, strain localization bands correspond to regions of localized strain that appear in both ductile (e.g. metals) and granular materials (e.g. geomaterials). From a mechanics perspective, this band formation constitutes a type of instability. Its origin has been debated on whether or not it is primarily related to geometrical, crystallographic or a combination of such effects [13]. For instance, it has been suggested that in fcc metals the band formation is preceded by the creation of obstacles to homogeneous deformation [13]. In addition, it was reported that the probability of banding increases when homogeneous dislocation slip is inhibited, as well as when insufficient crystallographic slip systems are available to accommodate the overall state of stress. In these cases, banding acts as a competing, non-crystallographic deformation mode [12], which justifies the need to investigate its role in the multiscale mechanical behavior of materials.

From an experimental point of view, plastic flow localizations have been recently investigated with the use of full field deformation measurements provided by Digital Image Correlation (DIC), as also shown by the authors [14], who first (to their best knowledge) demonstrated the connection between spatially varying twin activity and strain localizations in the form of bands in compression experiments of AZ31 magnesium alloy specimens. Later, Aydiner and Telemez [15] developed a multi-scale DIC setup to study the formation of shear bands on two orthogonal faces 
of AZ31B compression samples. They suggested that each compressive strain increment can be mainly accommodated by the emergence of a limited band, leaving the rest of the volume insensible to the externally applied load. Reported micrographs further showed the emergence of twins in these bands. Twin activity and associated strain localization were also reported by the authors to be associated with pronounced out-of-plane deformation profiles on the specimens' surface, a fact that was taken into account to modify the boundary conditions of a viscoplastic self-consistent crystal plasticity model coupled with continuous dislocation dynamics used to investigate the evolution of twinning during cyclic loading of Mg AZ31 [16].

To better understand the case of strain localizations in magnesium, it is well known that twinning has a profound effect on its mechanical behavior. Specifically, twin formation causes large reorientation $\left(\sim 87^{\circ}\right)$ of the crystal lattice, which manifests itself macroscopically by the sigmoidal stress-strain response dominated by a plateau before the strain-hardening region. In fact, it has been demonstrated that the near yielding behavior is governed by twin-related strain localizations that form macroscopically observed band-type patterns $[14,17]$. In this context, Changizian et al. [18] inspected the microstructure of an AZ81 alloy focusing on the contribution of twinning in accommodating the imposed strain and reported that the extensive twin formation observed was accompanied by strain localized in "micro-shear" bands. In fact, at early stages of deformation, several twin bands and mutual intersections of twins in a neighborhood of grains were reported. Due to the high misorientation angles associated with twinning, such twins ultimately act as barriers to overall dislocation movement within grains [19]. Therefore, it is also possible that dislocation pile ups cause high strain concentrations, which could lead to band formations. Furthermore, noticeable grain 
refinement inside band regions was observed which was attributed to the continuous dynamic recrystallization (DRX) occurring within these bands [20-22].

In this article, an integrated approach that combines testing and microstructure characterization results with a novel computational approach is presented. Emphasis is given on capturing the mechanistic effects of SLB formation, including the load drop at the onset of localization and the strain accumulation in the plateau region where crystallographic effects are important. To achieve these goals, a user-defined subroutine that combines plasticity with a stiffness degradation scheme is developed. Inputs for this model were obtained by the mechanical testing and characterization measurements.

\section{Methodology}

\subsection{Computational modeling approach}

The overall objective of the presented integrated method is to model macroscopically observed plasticity effects reported in magnesium specimens with SLBs, including the load drop at the onset of strain localization and the strain accumulation in the characteristic plateau of the stress-strain curve. To this aim, both 2D (plane stress) and 3D models were developed including notched, dogbone and hourglass type specimens as shown in Fig. 1, which were also (except the hourglass) tested experimentally. The reason for this variety of specimen geometries was to gain understanding on the effect of specimen shape and corresponding 3D deformation effects on the computed strain localizations and also to obtain direct inputs to the computational approach. 

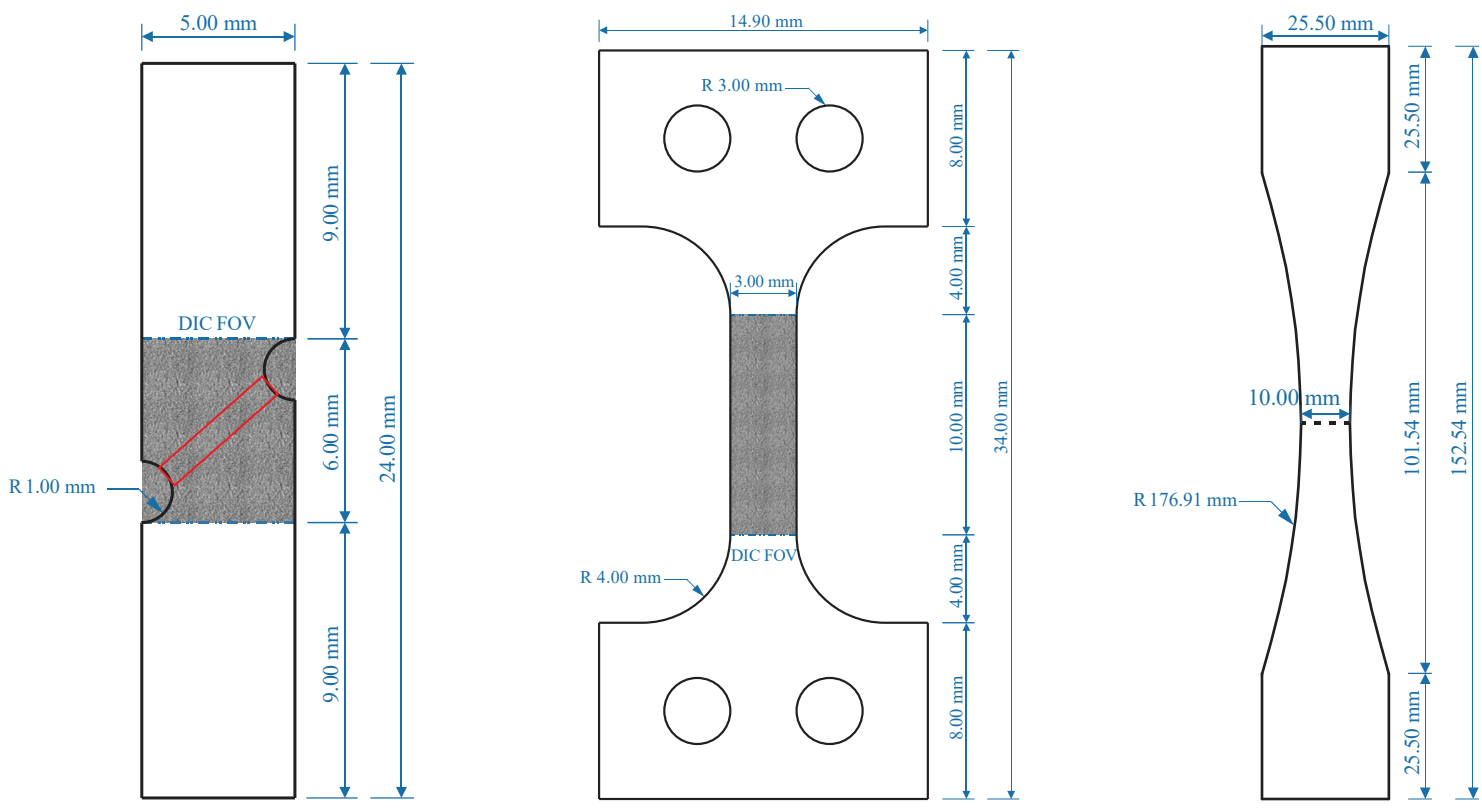

Fig. 1: Specimens geometry with marked FOV for DIC measurements in the notched and dogbone specimens. The hourglass geometry was used only for modeling

The computational part of this approach was implemented in a user subroutine (UMAT) in ABAQUS [23]. Specifically, actual experimental inputs were used in a user hardening (UHARD) subroutine. The plastic anisotropy in Mg alloys, manifested by the significantly different values of yield stresses measured depending on crystallographic texture, was considered in the numerical approach by directly using the corresponding stress-strain curves obtained experimentally. In addition, an isotropic hardening scheme was chosen as the plasticity model in which a stiffness degradation step was added to represent effects related to SLB formation. In the general case of biaxial or cyclic loading, a yield criterion that could account directly for plastic anisotropy and asymmetry effects could be introduced [see e.g. 24,25] as an extension of the method described herein. It should be noted here that the plasticity model adopted was purposely chosen to be agnostic to more complex descriptions of the behavior of magnesium alloys so that it can demonstrate the generality of the computational approach in this article in terms of simulating mechanistic effects of SLB formation. 
In Fig. 2a, a generic specimen under tension is depicted to better explain the strain localization effects targeted using the integrated approach in this article. At a certain stage of the applied displacement $u$ (or equivalently load), an instability in the form of a SLB is developed at an angle $\theta$ from the horizontal axis. Further increase of the applied loading results in an increase of the band's width $d$ as its fronts moves at a rate $\dot{y}$. Such material instabilities in the form of a band are triggered in this investigation through a stiffness degradation approach. Specifically, once an initiation criterion is met, the material stiffness is gradually reduced, creating a softened region. The value of the reduced stiffness in this region is obtained by the experiments described next, while this approach follows related modeling efforts $[10,26]$.

The yield function for the monotonic loading case considered in this investigation is defined by the following isotropic hardening model

$$
f=\left(\frac{3}{2} \boldsymbol{\sigma}^{\prime}: \boldsymbol{\sigma}^{\prime}\right)^{1 / 2}-r(p)-\sigma_{y}=0
$$

where $\boldsymbol{\sigma}^{\prime}$ is the deviatoric stress tensor, $r(p)$ is the isotropic hardening function, $\sigma_{y}$ is the yield stress and $p$ is the equivalent (accumulated) plastic strain, defined as

$$
p=\int_{0}^{t} \dot{p} d t, \quad \dot{p}=\left(\frac{2}{3} \dot{\boldsymbol{\varepsilon}}^{p} \dot{\boldsymbol{\varepsilon}}^{p}\right)^{1 / 2}
$$

where $\dot{p}$ is the equivalent plastic strain rate and $\dot{\boldsymbol{\varepsilon}}^{p}$ is the plastic strain rate tensor. Plastic strain increments were determined based on the following plastic flow rule

$$
\dot{\boldsymbol{\varepsilon}}^{p}=\frac{3}{2} \frac{\boldsymbol{\sigma}^{\prime}}{\sigma_{e}} \dot{p}
$$


where $\sigma_{e}$ is the effective stress, defined as

$$
\sigma_{\mathrm{e}}=\left(\frac{3}{2} \boldsymbol{\sigma}^{\prime}: \boldsymbol{\sigma}^{\prime}\right)^{1 / 2}
$$

The elastic strain and stress increments were also determined and all field quantities (stress components, effective plastic strain) were updated at the end of each increment.

Furthermore, a criterion was implemented to initiate the SLB. Specifically, the accumulated plastic strain (defined in Eq. (2)) was compared to an a priori defined critical value $\varepsilon_{c r}^{p}$ which could be either arbitrary (i.e. user-provided) or measured using experiments. If this critical value is reached or exceeded, a stiffness degradation approach was followed. Specifically, for those elements that exhibit a higher accumulated plastic strain, the corresponding stiffness matrix $\mathbf{C}$ and applied load (stress) $\boldsymbol{\sigma}$ is reduced according to the following relations:

$$
\mathbf{C}=(1-D) \overline{\mathbf{C}}, \quad \boldsymbol{\sigma}=(1-D) \overline{\boldsymbol{\sigma}}
$$

where $\overline{\mathbf{C}}$ is the initial element stiffness, $\overline{\boldsymbol{\sigma}}$ is the effective ('undamaged') stress tensor and $D$ is an overall (scalar) damage variable. The stiffness and load reductions alter the local stress-strain response, as described in Fig. 2b (top). Specifically, the 'undamaged' response is denoted with a dashed line and the 'damaged' path is displayed with a continuous one. In this approach, the damage variable is expressed as a function of accumulated strain given by the expression

$$
D(\varepsilon)=d_{f} \cdot \varepsilon^{a}
$$

where $d_{f}$ and $a$ are a degradation factor and a coefficient to characterize damage progression, respectively. 
(a)

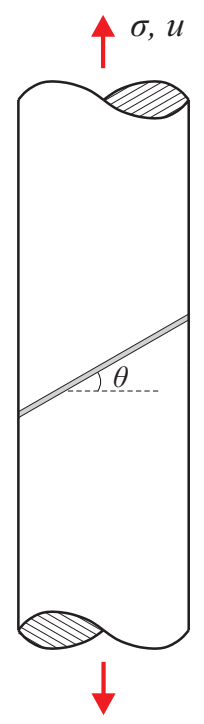

Shear band initiation $t=t_{0}$ (b)

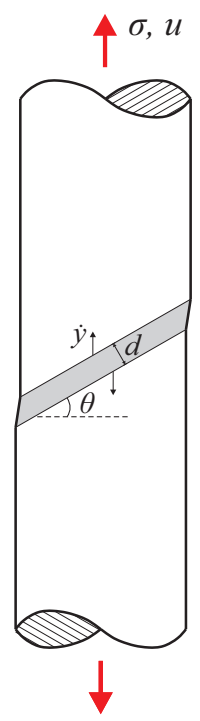

Shear band growth at time $t$
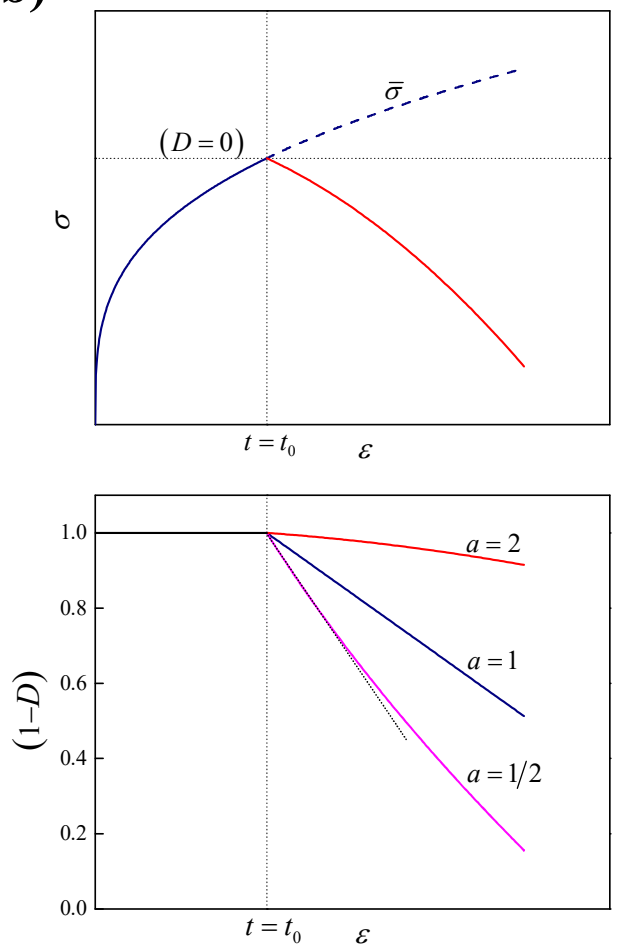

Fig. 2: (a) Schematic of shear band initiation and growth geometry, (b) stiffness degradation scenarios.

In Fig. 2b (bottom) different stiffness degradation scenarios are depicted. The reduction rates shown in Fig. 2b (bottom) could be further connected to the underlying microstructure evolution, actual twin formation characteristics including spatial distribution and volume fraction) through a phenomenological description, as for example in Barnett et al. [17].

After the criterion is examined at each integration point, the material tangent stiffness matrix is calculated and returned to the finite element solver. An implicit integration scheme based on the radial return method was adopted for the purpose of this work. This scheme begins with a selection of a trial stress increment and the determination of a trial yield function. If yielding does not occur $(f<0)$, the algorithm updates all field quantities and returns them to the solver along 
with the consistent Jacobian. If yielding occurs $(f>0)$, the effective plastic strain increment was identified through a Newton's iteration scheme. A subroutine (UHARD) was used to calculate the hardening for the current yield state based on tabulated stress-plastic strain experimental data imported into the code. For the $i$-th increment, the hardening is evaluated as

$$
r^{i}(p)=\frac{\sigma_{y}^{i}-\sigma_{y}^{i-1}}{\varepsilon_{i}^{p}-\varepsilon_{i-1}^{p}},
$$

where $\sigma_{y}^{i}, \varepsilon_{i}^{p}$ and $\sigma_{y}^{i-1}, \varepsilon_{i-1}^{p}$ are pairs of yield stress and plastic strain values at the current and previous increments respectively.

The overall computational approach is described in the form of a pseudocode in Fig. 3. This numerical procedure was successfully implemented in ABAQUS [23] as a user subroutine, suitable for both $2 \mathrm{D}$ and 3D problems. It should be mentioned that due to the nonlinearities introduced, the problem is either solved statically or dynamically with the aid of (artificial) damping. The 2D models comprised approximately 50,000 degrees of freedom (DOF), using quadrilateral linear plane stress elements (CPS4) and an average mesh size of $0.5 \mathrm{~mm}$. The 3D models consisted of $\sim 400,000 \mathrm{DOF}$, with an average mesh size of $0.5 \mathrm{~mm}$ and linear eight-node brick elements (C3D8). 


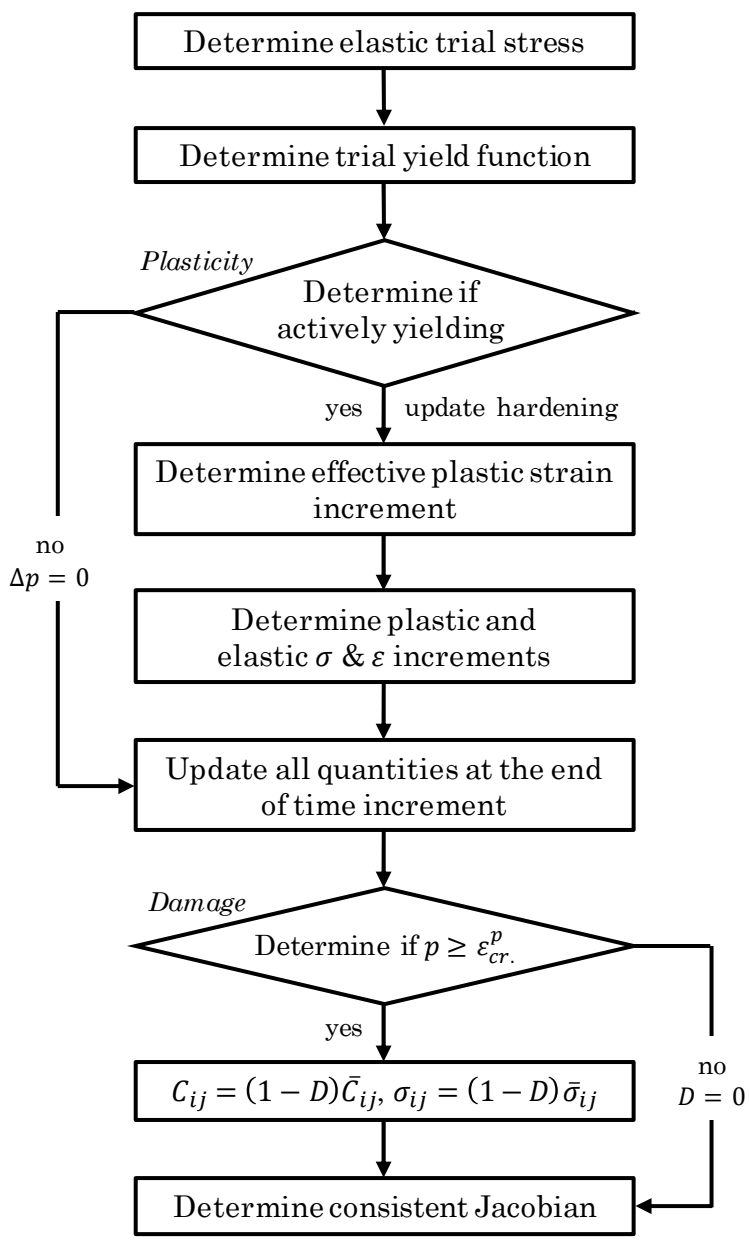

Fig. 3: Pseudocode for shear band realization in a finite element code

The boundary conditions used in all simulations are described in Fig. 4a. The top and bottom ends were assumed rigid in accordance with the actual experimental conditions. Hence, the samples were fixed at the top rigid area while displacement was applied at the bottom. An additional region was identified in the hourglass and notched specimens that corresponded to the area defined by two parallel lines set at a distance of $10 \mathrm{~mm}$ from the midpoint, where localization in the form of either necking or SLB was expected to occur due to geometry. In the case of the notched specimen this region was defined based on the FOV in Fig. 1. 
A mesh sensitivity study was conducted before the implementation of the stiffness degradation step and pertinent results are shown in Fig. 4b. The objective of this study was to identify the mesh size that yields convergent results based on some metric and for a given element type. In general, mesh dependency in similar localization problems is a critical parameter to be evaluated as reported in the pertinent literature $[6,27,28]$. In the case of the hourglass specimen, the inset in Fig. $4 \mathrm{~b}$ shows the region where the computed equivalent plastic strain at a selected stage of the analysis (which corresponded to 5\% applied strain) was averaged in the partitioned region at the middle of the specimen for three types of elements (linear tetrahedral, linear and quadratic hexahedral) and for several mesh densities. Convergence was achieved at $\sim 2.4 \times 10^{5}$ degrees of freedom (DOF) for linear tetrahedral and linear hexahedral elements, and at $\sim 4.5 \times 10^{5}$ DOF for quadratic hexahedral elements. The corresponding average element sizes for convergent solution were equal to $0.6 \mathrm{~mm}$, $0.6 \mathrm{~mm}$ and $0.8 \mathrm{~mm}$ respectively.

It was further observed that with the application of the criterion the problem under investigation was dependent not only on the mesh size but on the mesh structure as well. Specifically, since the proposed modeling approach does not rely on any imperfection to introduce a strain localization zone, as frequently employed in similar efforts previously reported [7,2], the mesh structure could affect the location of SLB initiation. To address this issue, structured meshes were used following similar work in the literature $[10,26,7]$. In fact, it was observed that even slight mesh nonuniformities had an effect on the initiation location of strain localizations. Consequently, the mesh type used is shown in Fig. 4c, which corresponds to the notched specimen in Fig. 4a. For the models 
in Fig. 4c, two element sets are shown, one that corresponds to the FOV used to compute the strain in the SLB and an additional one that corresponds to the larger FOV, as also shown in Fig. 1.

(a)

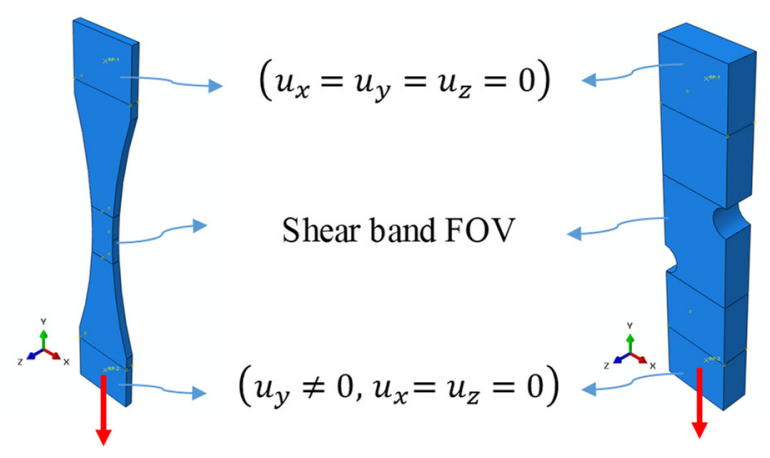

(b)

(c)
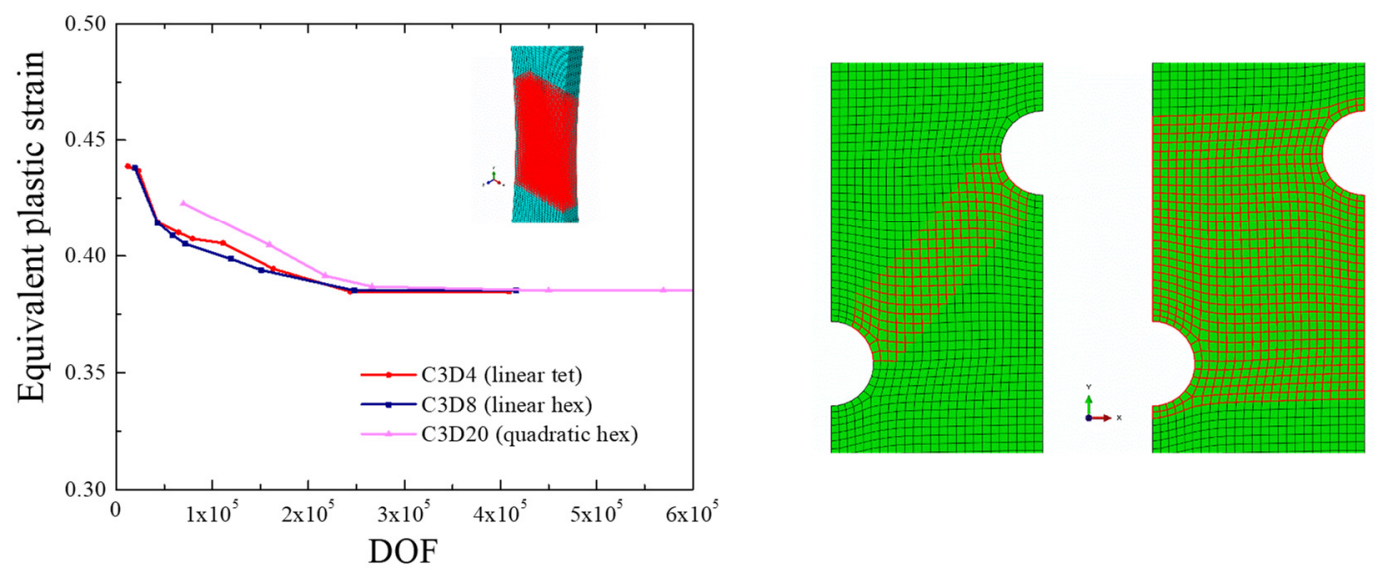

Fig. 4: (a) Boundary conditions and loading assignment description for the two geometries considered, (b) mesh sensitivity study for an hourglass specimen, (c) shear band and full field of view definition in the notched specimen

\subsection{Experimental inputs to the computational approach}

Experiments using a commercial magnesium AZ31 alloy were conducted to obtain inputs to the computational approach in this investigation. Details on the specimen preparation procedure are given in the Appendix. Electron backscatter diffraction (EBSD) data before deformation are shown in Fig. 5, demonstrating crystallographic alignment predominantly parallel to the ND direction for specimens cut perpendicular to the rolling direction (called ND samples), as well as texture 
predominantly perpendicular to the RD direction resulting to specimens that are called TD, since they are cut in the TD direction. Note that the $c$-axis is aligned with the ND direction for the ND samples, while it was perpendicular to the RD direction for the TD samples. Hence, extension twinning was expected to occur in tensile testing of the ND samples only.

Given the achieved microstructures and corresponding specimens, a number of inputs to the computational modeling based on experiments were attempted, as explained next in this section.
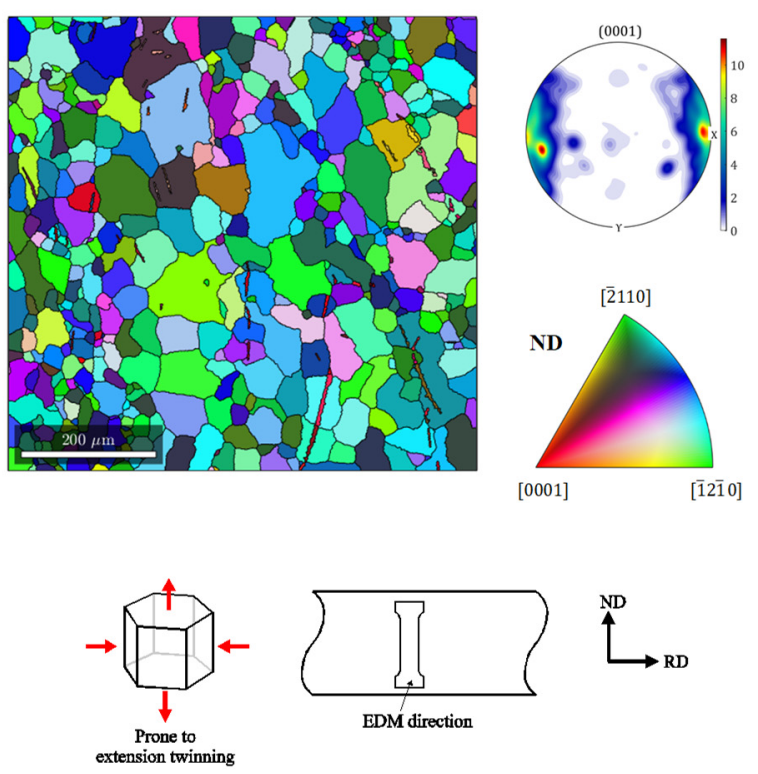
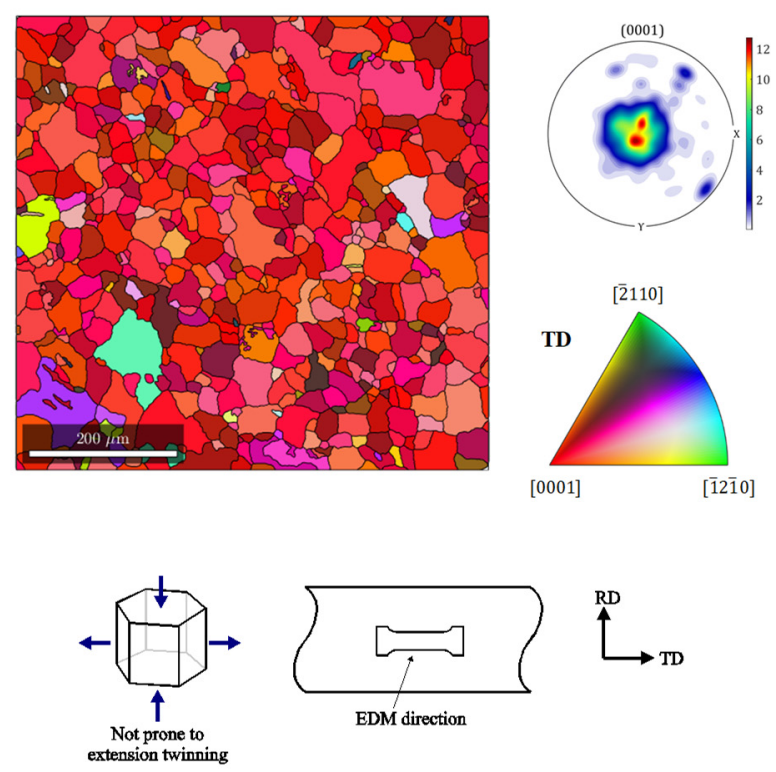
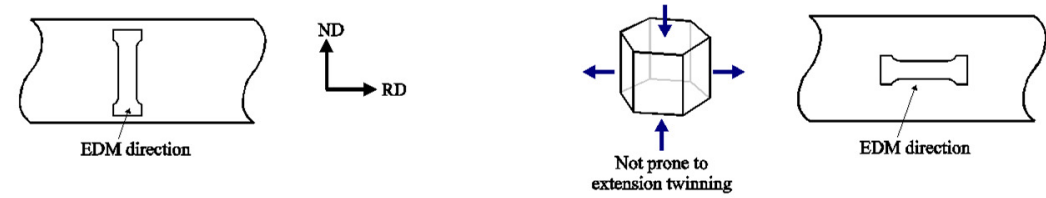

EDM direction

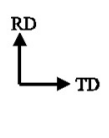

Fig. 5: Texture and specimen information for the experimental portion of this article

The double-notched geometry (Fig. 1a) was used to provide an a priori defined strain localization zone and assist the strain quantification in the SLBs. Details on the mechanical testing and DIC parameters are given in the Appendix. Fig. 6 shows the obtained stress-strain curves for the two textures defined in Fig. 5, in addition to snapshots of the full field strain field along the loading direction ( $y$-axis in this case) at four different time instances, displayed for the ND (top row) and 
TD (bottom row) specimens, respectively. A fixed range legend was used so that the initiation and growth of the SLBs become more evident both as a function of the bulk strain increment as well as its geometry. For the stress-strain curves the average full field true strain $\bar{\varepsilon}_{y y}$, as shown in the snapshots of Fig. 6, was computed by the DIC method.

As seen in Fig. 6, a strain localization zone is formed on the ND specimen. This zone initiated at relative low bulk strain levels $\left(\bar{\varepsilon}_{y y}=0.0028\right)$ compared to the TD specimen $\left(\bar{\varepsilon}_{y y}=0.0044\right)$. The strain localization in the TD specimen, which is not favorable to twinning, appears to develop in a less geometrically ordered way compared to the almost contained within the band strain localization seen in the ND tension sample, which is twin favorable. Hence, the formation of a pronounced SLB in ND specimens could be related to the formation of twinning. In fact, it has been reported [29] that when a propagating twin interacts with a grain boundary (or other defects e.g. a stacking fault), high stress concentrations may develop and as a result twin nucleation may take place [30]. Such induced twinning near grain boundaries was also reported by the authors before, and was related to both Schmid factor and dislocation stored energy effects [14,16]. In addition, since twins can locally and "autocatalytically" generate further twinning, it was reported earlier by the authors that "twin walls" can be formed which were also found to be correlated with strain localization patterns $[14,17]$. Microstructure evidence on twinning is shown later in this section.

To quantify such strains inside the localized regions shown in Fig. 6 and make comparisons between the two types of textures, the available DIC data were used to compute strain values for two separate fields of view, schematically shown in Fig. 1, consisting of one inside the shear band (diagonally between the two notches marked with the red rectangle in Fig. 1) and one in the entire 
area shown in Fig. 6 (with dimensions specified in Fig. 1). The resulting average values for both FOV are presented in Fig. 7 by the ratio of $\bar{\varepsilon}_{y y}^{\text {SB FOV }} / \bar{\varepsilon}_{y y}^{\text {Full FOV }}$ corresponding to the computed strain values for both ND and TD specimens. Interestingly, although only qualitative differences can be made by visual comparison of the data in Fig. 6, the results in Fig. 7 demonstrate that the average longitudinal strain in the SLB region of the ND specimen quickly rises up to a maximum value of 2.7 times that of the average strain of the sample (at $\bar{\varepsilon}_{y y}=0.40 \%$ ), while the corresponding value for the TD specimen reaches a peak of only 1.87 at a much later state of specimen deformation (at $\left.\bar{\varepsilon}_{y y}=1.88 \%\right)$. In fact, the ratio of 1.87 is attained in the ND case at approximately the same strain level $\left(\bar{\varepsilon}_{y y}=1.84 \%\right.$ ) compared to the TD specimen; hence it is probably geometry determined.
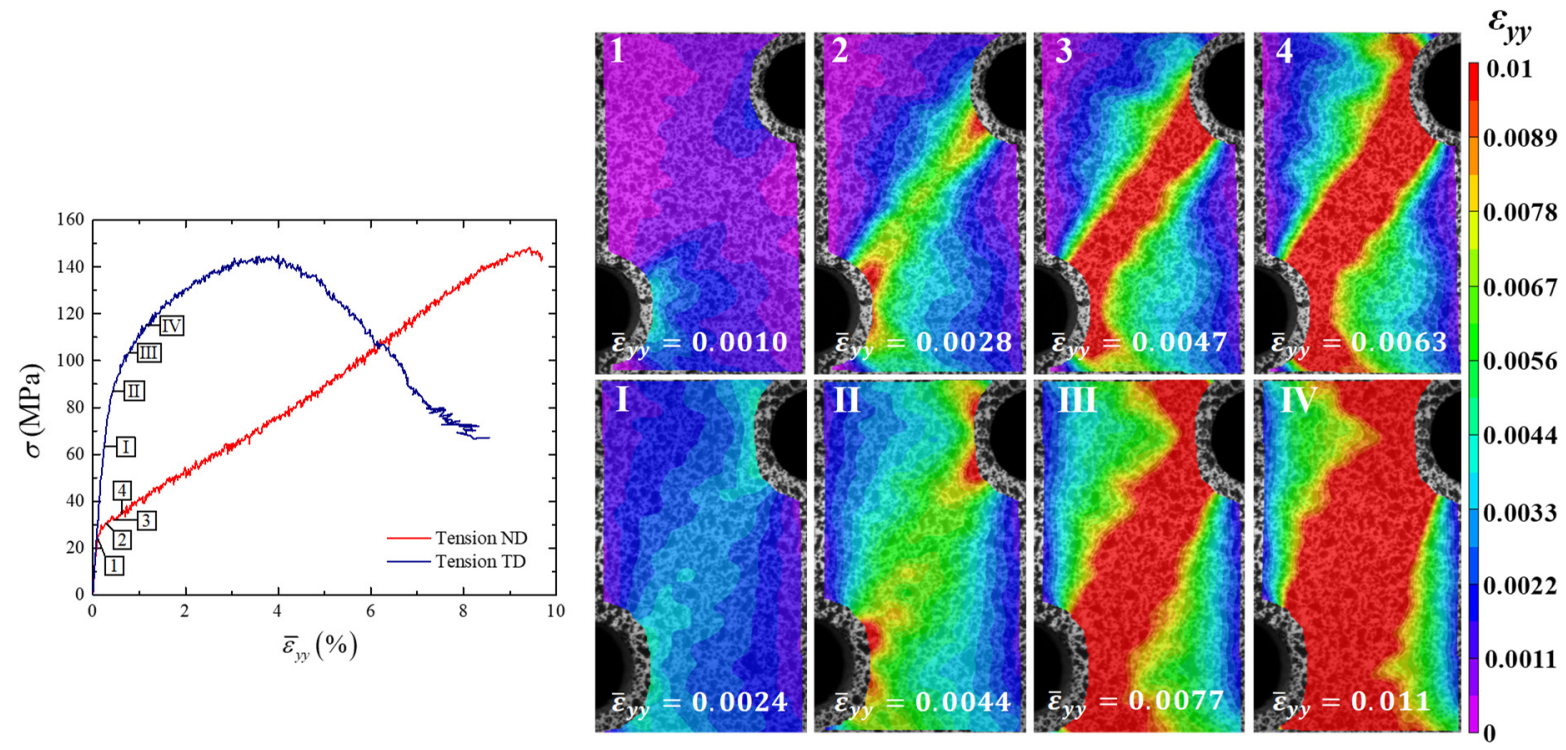

Fig. 6: Strain localization evolution for ND (top row) and TD (bottom row) specimens

Given the findings in Fig. 7, it could be stated that the sharp increase of the strain in the

SLB of the ND specimen happens relatively early in the deformation process and at overall strain 
that the mechanical data in Fig. 6 show to be related to the onset of yielding, which has repeatedly been associated with twin nucleation and growth. In addition, the fact that the ratio in the ND case appears to exponentially drop past the peak value of 2.7 can be explained by the fact that as the overall strain in the specimen increases, the strain in the entire FOV measured in Fig. 6 also increases. Hence, such an increase is mostly due to the localization induced by the adopted notched geometry which is common between the ND and TD specimens. Consequently, the results in Fig. 7 are considered as a first indication of the effect that the strain in the SLB region has on the overall plasticity of such magnesium specimens.
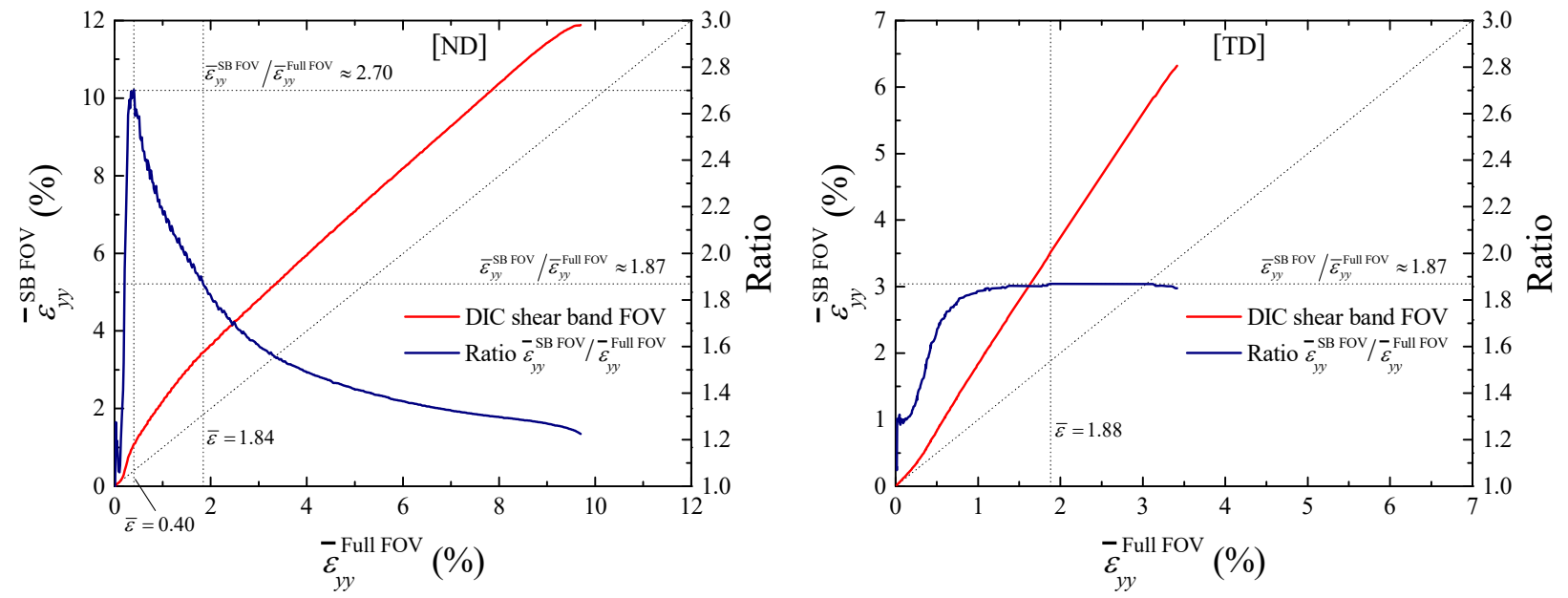

Fig. 7: Ratio between average strain in the SLB and full FOV for ND and TD notched specimens

To reduce the geometry effect in the notched samples, a straight dogbone specimen (Fig. 1b) was also tested. The details of the experimental setup can be found in the Appendix. Figure 8 shows the stress-strain strain curve from two samples machined in different directions (in accordance with Fig. 5). The stress-strain curves obtained match the corresponding mechanical behavior of magnesium alloy and its reported plastic anisotropy. In addition, DIC strain maps of selected early 
strain stages from both ND (left) and TD (right) samples with fixed legend are shown to illustrate the growth of SLB in the ND compared to the TD specimen. As seen in Fig. 8, the strain localization became noticeable at very low strain $\left(\bar{\varepsilon}_{y y}=0.20 \%\right)$ in the ND specimen while no localization is observed at the same strain increment in the TD specimen. Moreover, while at $\bar{\varepsilon}_{y y}=0.54 \%$ the maximum strain in the SLB of the ND sample was found equal to $\bar{\varepsilon}_{y y}^{\max }=1.50 \%$ the TD specimen had a much smaller peak $\left(\bar{\varepsilon}_{y y}^{\max }=1.05 \%\right)$ at a different location.
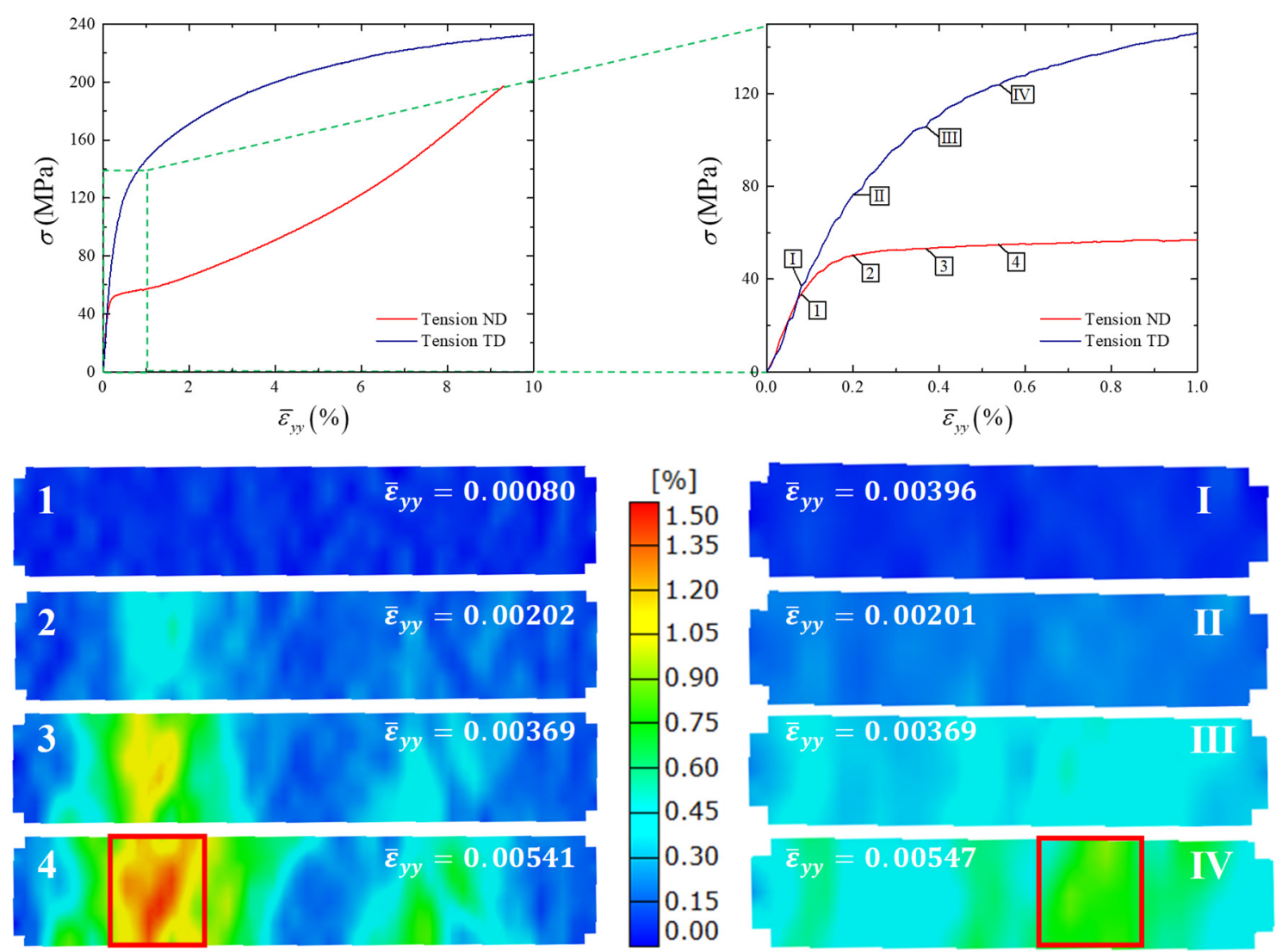

Fig. 8: Strain localization evolution for ND (left) and TD (right) specimens

The ratio computed and reported in Fig. 7 is also shown in Fig. 9 for the dogbone specimens of Fig. 8. Interestingly, the straight ND specimen exhibits a similar behavior as the notched one. 
Specifically, the average strain in the SLB region (marked with the red box) rises to 2.51 times more than the average strain (at $\bar{\varepsilon}_{y y}=0.35 \%$ ) in ND specimen, while the corresponding ratio in TD sample peaks at 1.68 (at $\bar{\varepsilon}_{y y}=1.12 \%$ ). The maximum value of localized strain in the ND specimen drops much faster compared to the corresponding value in the notched sample. This could be attributed to the fact that the notched specimen affects the location and growth of SLBs, while in the straight specimen such localizations can propagate freely. Nevertheless, the initiation of the SLB matches well the one observed in the notched specimen, providing strong evidence that it might be caused by microstructural effects, which is explored next.
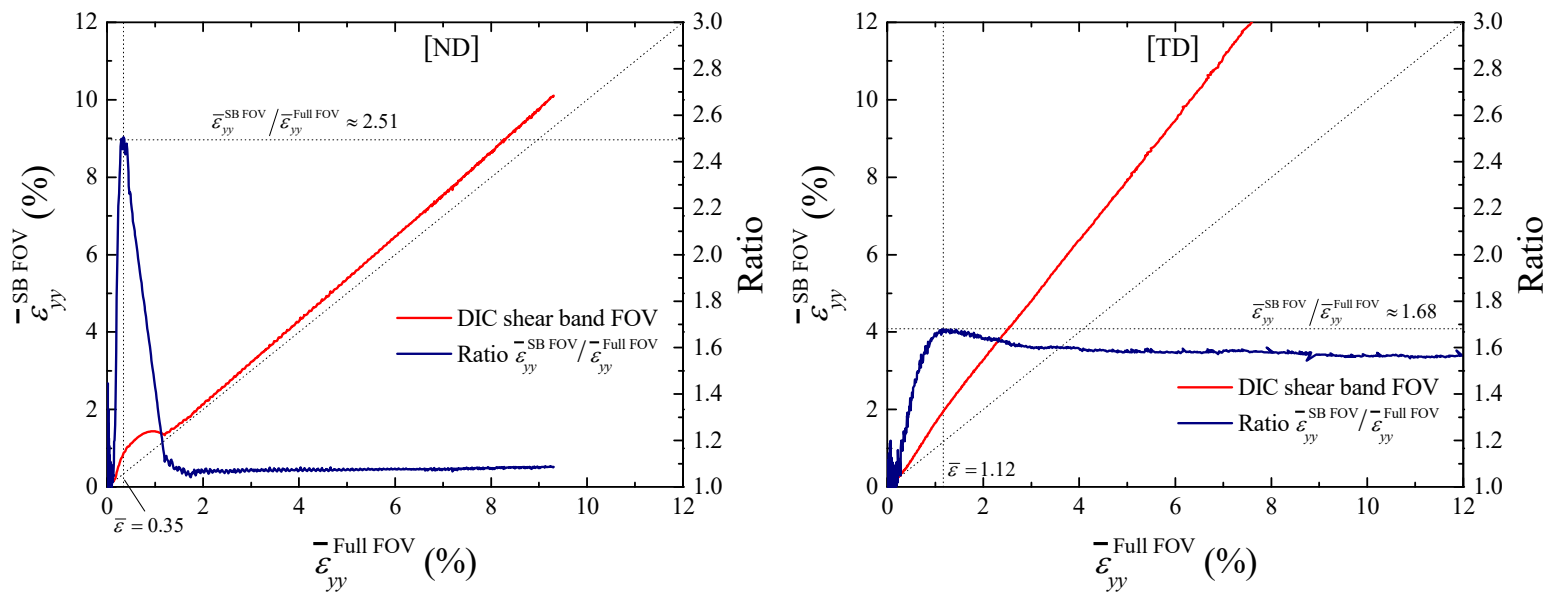

Fig. 9: Ratio between average strain in the SLB and full FOV for ND and TD dogbone specimens

To investigate the origin of strain localizations, post mortem microscopy was performed on both types of specimens. Several twins were found throughout the SLB of the ND notched specimens, as shown in Fig. 10a. Such twinning activity could be thought of as a response to local strain accommodation since not all grains in the area with macroscopic strain localization was found to be twinned. In this case, the observed twins were found, as shown in Fig. 10b to extend past several 
grains within areas of the SLB, which agrees with previous findings by the authors [14] and provides further evidence on the role of twinning in strain localization of magnesium alloys. Twin boundaries are highlighted in Fig. 10a and 10b, while the misorientation angle was found to be between $86^{\circ}$ and $87^{\circ}$ which is in agreement with previous work. This further corroborates the findings reported by Barnett et al. [17], Beyerlein et al. [31], and Hazeli et al. [14]. On the other hand, for the TD texture, the EBSD scan performed after loading showed no indication of twinning, as expected. It is important to note that the cross-boundary twinning activity reported herein appears to follow the direction of the band front. This observation can therefore be regarded as evidence of twinning acting as a barrier to the motion of the SLB front, which results in progressive reduction of the front's rate of growth (denoted as $\dot{y}$ in Fig. 4); it could subsequently also lead to an increase of strain levels inside the band.

(a)

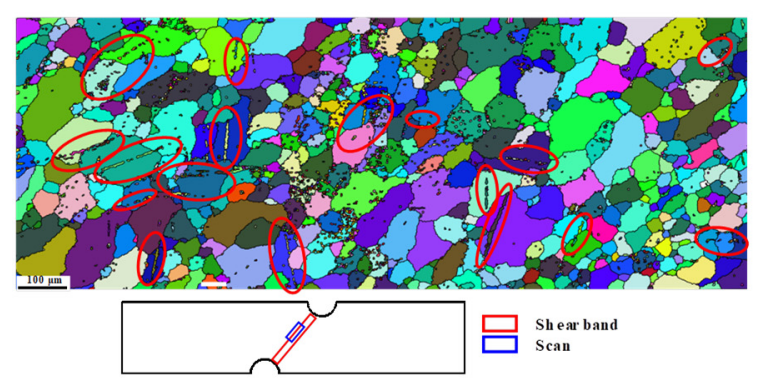

(b)

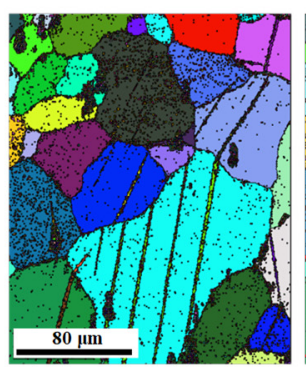

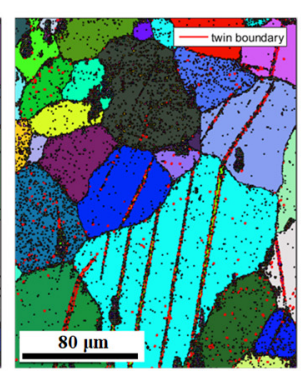

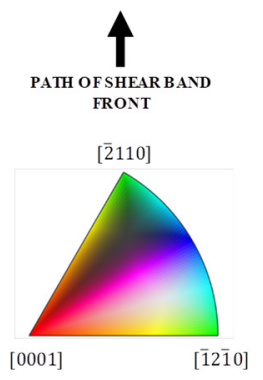

Fig. 10: (a) EBSD-IQ scan around the edge of SLB region for the ND specimen (b) extension twins

Similar evidence is also found in the straight ND specimen. A separate interrupted test within the Scanning Electron Microscope was conducted with in-situ EBSD providing microstructure evolution in the plateau region. Four EBSD scans were taken at a randomly selected location for four strain values, as shown in Fig. 11. The twin area fraction, defined as the number of grains 
that have been twinned over the total number of grains, grew during the plateau region while a large jump between $\varepsilon_{2}$ and $\varepsilon_{3}$ was observed. At $\varepsilon_{3}$, the area monitored revealed extensive twinning activity with lots of cross-boundary twins similar to previous results shown in Fig. 10. Moreover the jump in the twin area fraction is accompanied by a minimum value of stiffness as quantified by the derivative shown in Fig. 11. This minimum indicates that softening occurs due to texture changes caused by changes in the rate of twinning as quantified by the twin area fraction. After $\varepsilon_{3}$ work hardening seems to start affecting the overall deformation.
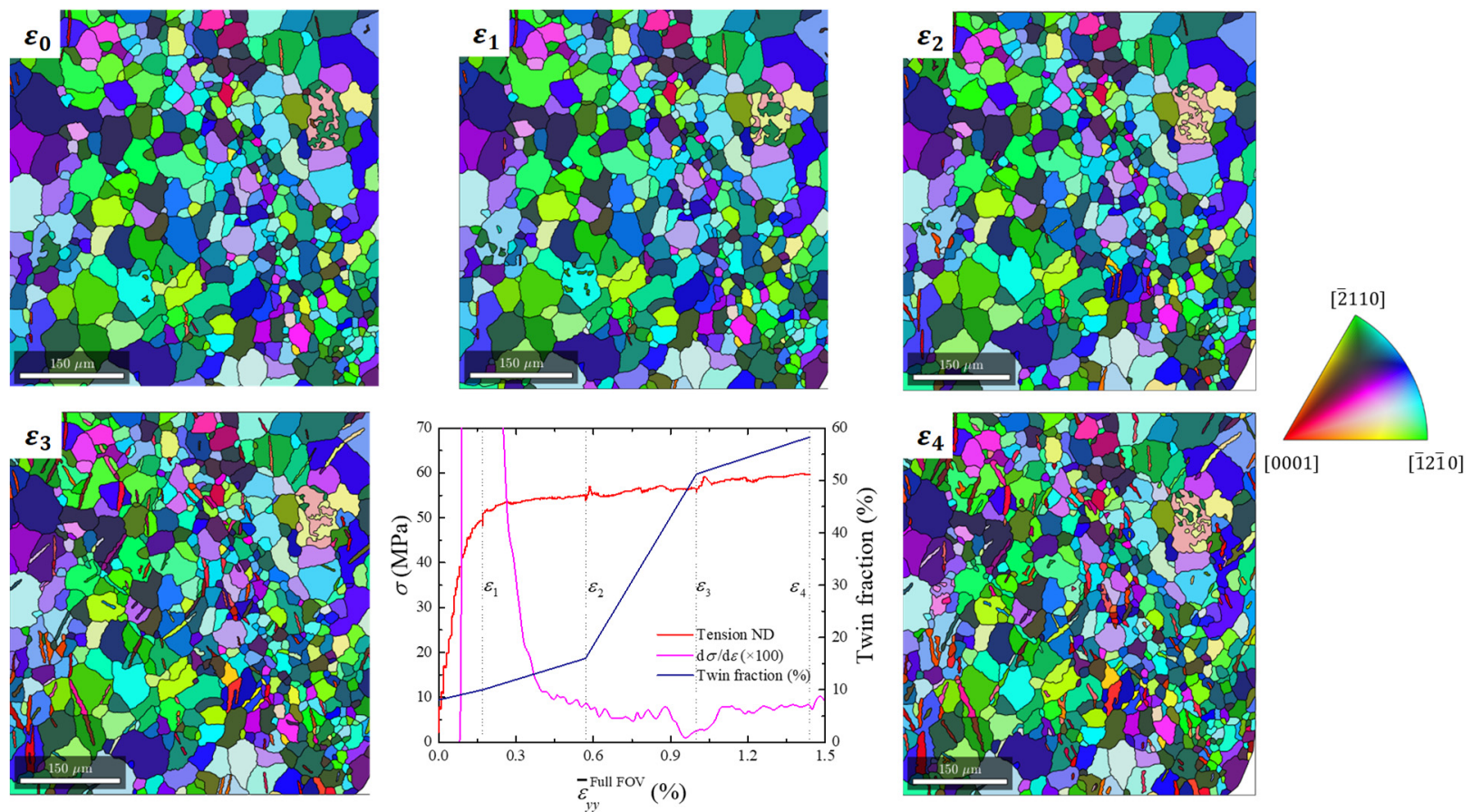

Fig. 11: EBSD-IQ scans at different stages of applied loading and corresponding stress-strain response along with twin fraction and rate of change of stress over strain

\subsubsection{Load drop at the initiation of the $S L B$}

The critical strain value for stiffness degradation $\varepsilon_{c r}^{p}$ defined in the computational approach section was determined from the DIC results obtained for dogbone specimens. Specifically, load 
serrations attributed to dislocation interactions have been reported in the literature that could potentially cause Lüders bands near yielding [32]. Moreover, significant load drops have been observed by the authors in magnesium single crystal compression testing due to the initiation of strain localization [33]. Taking both these remarks into account, the force vs time response is plotted in Fig. 12 and is compared to the first derivative over time, as load drops are expected to trigger changes in the first derivative.
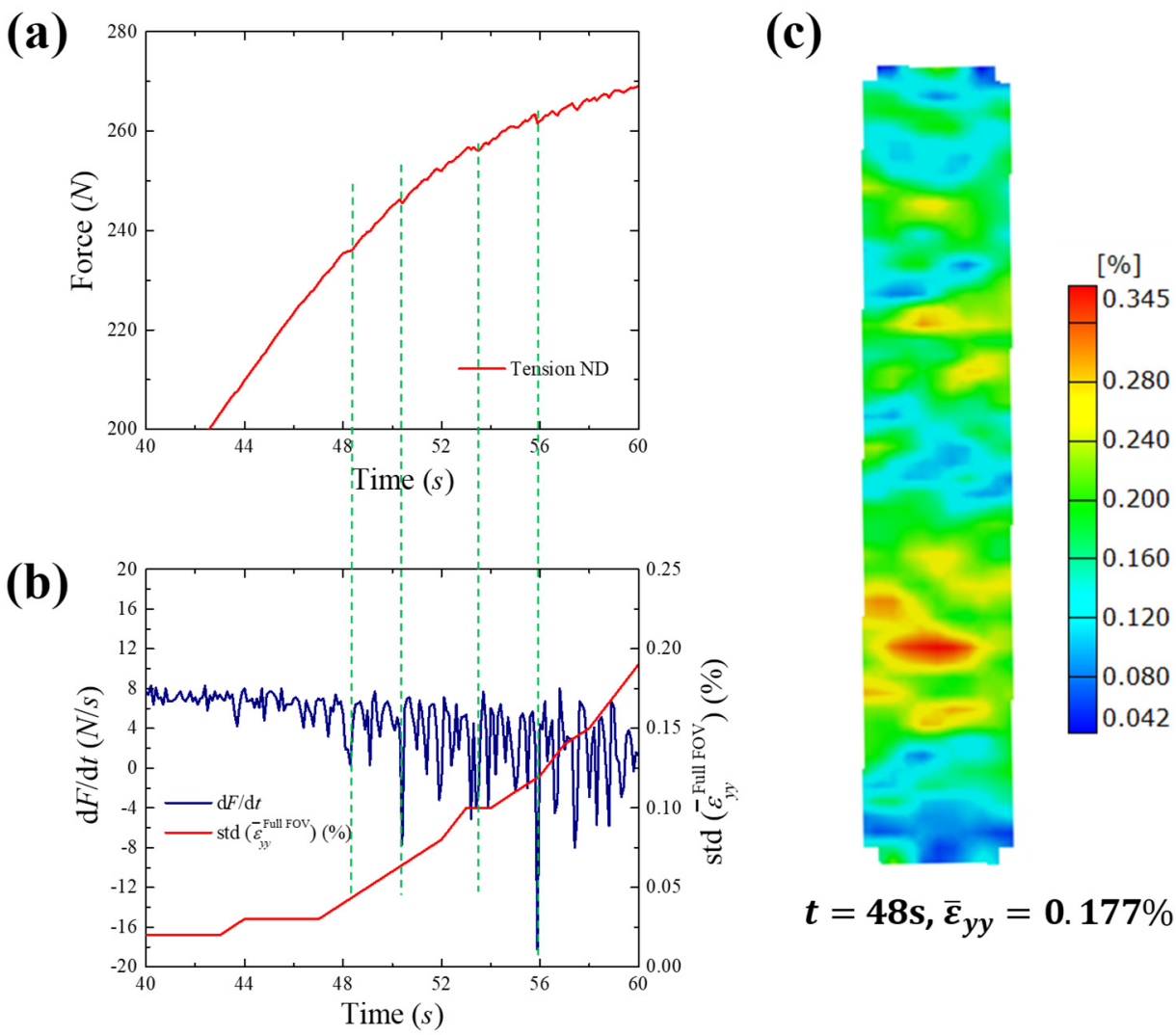

Fig. 12: (a) Force vs time plot for a straight ND specimen; (b) evolution of the first derivative of the force over time and the standard deviation of the average strain in the FOV; (c) corresponding full field plot at initiation time

In addition, the standard deviation of the strain values inside the FOV was also calculated for each time instance. The occurrence of SLB is expected to increase the standard deviation as the 
increased strain in the SLB would deviate from the average value in the FOV. In fact, and as seen in Fig. 12, load drops are accompanied by changes of the derivative and jumps in the standard deviation. This provides convincing evidence that a good estimate of the critical strain value to be used in modeling could be $\varepsilon_{c r}=0.177 \%$. Actually, the DIC snapshot at this time instance is also shown in Fig. 12c and demonstrates the initiation of a SLB at the bottom half of the specimen.

\subsubsection{Stiffness degradation quantification}

After identifying the critical strain for damage initiation, the stiffness degradation in the plateau was also attempted to be quantified from the conducted experiments. In fact, it was reported previously that stiffness drops due to texture changes caused by deformation twinning [34]. To measure this softening response, a separate fully reversed cyclic loading of the straight ND sample was conducted and the obtained stress-strain curve is shown in Fig. 13. Specifically, the specimen was loaded until within the plateau region. An estimate of the specimen's stiffness in this region was obtained by computing the slope in the unloading portion and was found to be equal to 13.9 GPa, which agrees with previously reported values and is considerably less than the stiffness measured during tensile loading confirming the softening effect. 


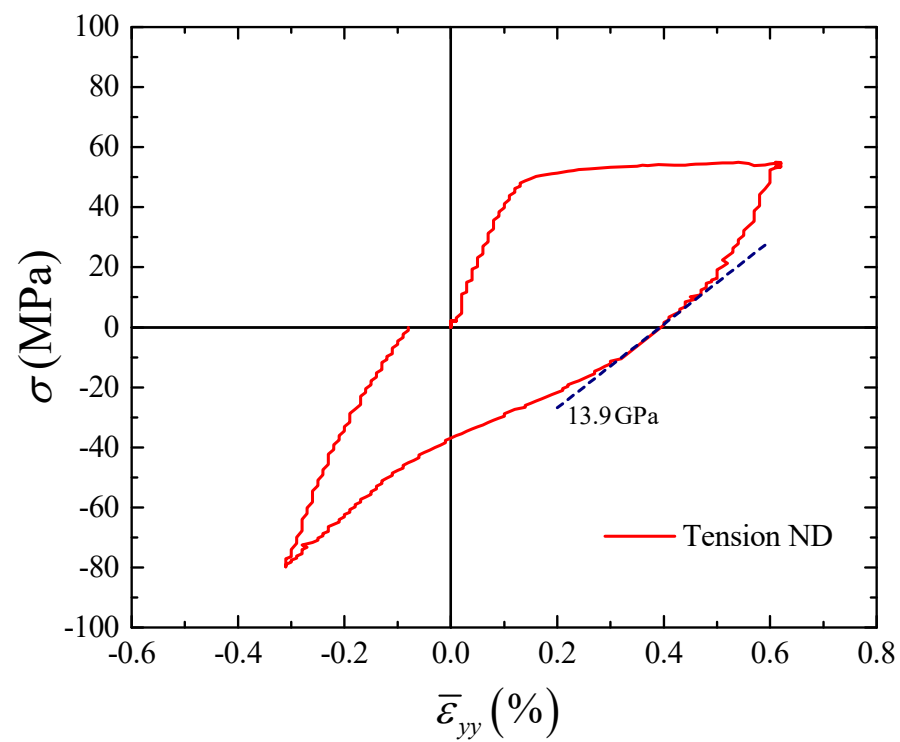

Fig. 13: Loading-unloading process to evaluate the stiffness in the plateau region

\section{Results and discussion}

\subsection{Strain inside the $S L B$}

The objective of the modeling approach was to create a methodology to compute stain localizations and relate them to global effects, similar to the experimental portion of this investigation. Several geometries were computationally tested to understand geometrical contributions to localized deformations in addition to effects caused by the adopted modeling approach. Specifically, the double notched and the hourglass specimens were used in FEM simulations. The height of the hourglass specimen was $152.54 \mathrm{~mm}$, its thickness equal to $3.18 \mathrm{~mm}$ while the minimum width was $10 \mathrm{~mm}$ and the arc length $176.91 \mathrm{~mm}$ (see also Fig. 1).

Computational results for the case of the notched specimen are shown in Fig. 14. Specifically, in Fig. 14a stress-strain curves are shown for both ND and TD specimens calculated by using strain averages computed in the larger FOV, as described in Fig. 4c. The response of the TD and ND 
specimens numerically computed agrees with the available experimental data (Fig. 6), which was expected as actual stress-strain curves were used to define the plasticity in these modes. The response of the TD specimen for which in addition to plasticity, a strain-based stiffness degradation initiation criterion of $\varepsilon_{c r}$. $=0.177 \%$ was used is also plotted in this figure (denoted as 'damaged' as opposed to 'undamaged' which did not employ such criterion) to demonstrate the capability of the approach to affect global and local strain values. The objective of studying a 'damaged' TD specimen was to demonstrate the way the softening criterion can alter an arbitrary nonlinear hardening material law, so that it could model the mechanistic contribution that could be caused by the sudden activation of a plasticity mechanism, such as twinning in this case. The parameters $d_{f}$ and $a$ were set equal to 1.98 and 0.01 , respectively, in order to match the softening response of Fig. 13. The calibration of these parameters was found to be a non-trivial task because the initiation criterion, the stressstrain response, and twin evolution taken from experiments were actually based on average values obtained by full field results either of DIC or EBSD of a certain area. This information is computationally imposed at a finite element level with the outputs being averaged again over a given FOV. Therefore, the parameters $d_{f}$ and $a$ are selected in order to match the experimentally observed macroscopic response. Depending on the mesh discretization, the damage variable evolution decreasing rate needs to be adjusted so that the softened region would propagate spatially in an appropriate way for the average stress-strain fields to match the macroscopic experimental values. 
(a)

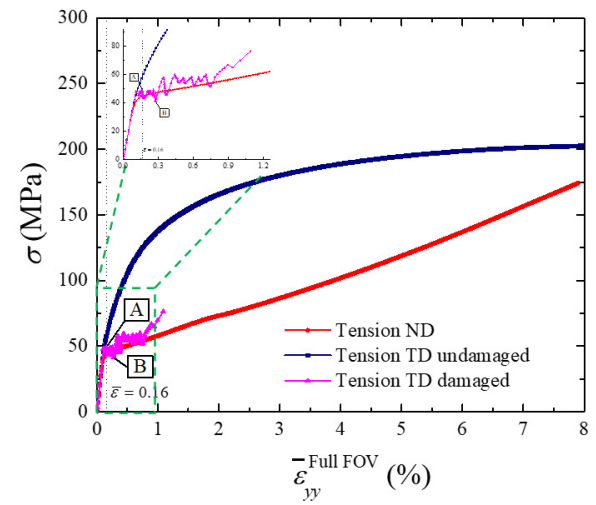

(b)

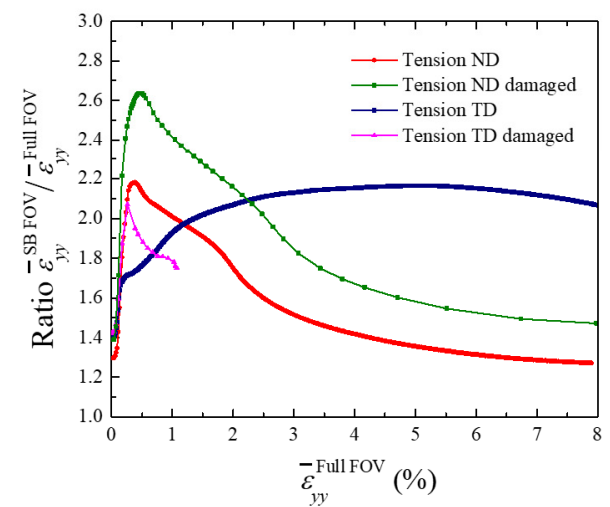

(c)
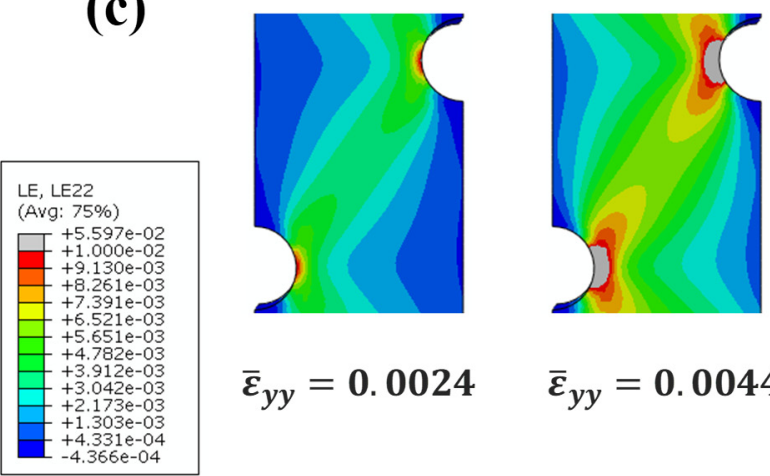

$$
\bar{\varepsilon}_{y y}=0.0024
$$

$\bar{\varepsilon}_{y y}=0.0044$

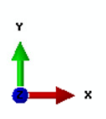

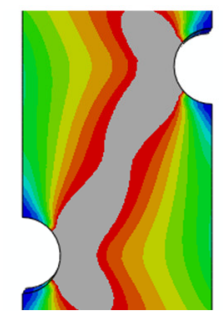

$\bar{\varepsilon}_{y y}=0.0077$

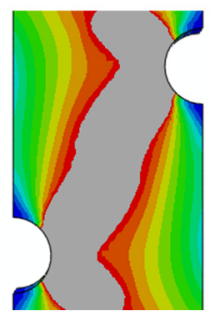

$\bar{\varepsilon}_{y y}=0.011$

Fig. 14: (a) Stress-strain curves for damaged TD and undamaged TD and ND specimens, (b) ratio between average strain in the shear band and full FOV region, (c) strain evolution TD damaged specimens

Based on the results presented in Fig. 14 it can be observed that the stiffness of the damaged specimen diverges from that of the undamaged at very early strain stages. This behavior is expected since some elements in these simulations reach the critical value selected during the initial loading stages. Consequently, their stiffness is degraded and as a result the global stiffness computed is also reduced with respect to the undamaged specimen. In addition, a stress drop was observed at $\varepsilon \approx 0.16 \%$ (point $\mathrm{A}$ ) leading to a local minimum at $\varepsilon \approx 0.28 \%$ (point $\mathrm{B}$ ) followed by a subsequent increase of stress as a function of average strain in the loading direction. This type of local decrease followed by an increase in the stress-strain curve is representative of a shear band formation in similar computational efforts reported in the pertinent literature (see e.g. $[35,8]$ ). 
To further characterize the strain effects in these simulations, the average strain values were extracted from the two element sets defined in Fig. 4c and in accordance with the results in Fig. 7 and Fig. 9. The ratio of the average strain in the two regions, defined again as $\bar{\varepsilon}_{y y}^{\text {SB FOV }} / \bar{\varepsilon}_{y y}^{\text {Full FOV }}$ is shown in Fig. 14b, as a function of the average strain in the full field region. The general trend computationally obtained is similar to the experimental results in Fig. 7; the maximum ratio value computed in the ND specimen was equal to 2.18 (as opposed to 2.7 measured by the DIC data) and was achieved at $\bar{\varepsilon}_{y y}=0.39 \%$. The same ratio in the (undamaged) TD specimen is also in good agreement with the experimental results, showing a peak value of 2.16 much later in the deformation history ( $4 \%$ ). Interestingly, the response of the damaged TD specimen resembles the behavior of the ND specimen in the early strain stages, providing evidence on the effect of the adopted plasticity and damage initiation criterion on global trends. Indeed, there is a distinct maximum value of 2.07 at $\bar{\varepsilon}_{y y}=0.26 \%$ (which is very close to the value of $0.4 \%$ measured experimentally) followed by a monotonically decreasing response. On the other hand, application of the damage criterion on an ND specimen improves the response, as the maximum ratio value computed was 2.64 , which is very close to the DIC value. However, the strain value in the SLB is overestimated as further deformation is applied, since the ratio does not drop as quickly compared with the experimental plots (Fig. 7).

Regarding the local aspect of these results, strain results are presented for the damaged TD case in Fig. 14c at four different loading instances which were selected so that the average strain levels in the chosen FOV are the same as the DIC results in Fig. 6. A fixed colormap was also used to track the initiation and development of strain localizations. In general, a direct comparison of 
Fig. 6 with Fig. 14c shows that the softening criterion spatially alters the computed strain localizations, as the shear band thickness for the damaged specimen appears to be reduced compared to the undamaged case (not shown but related results are shown in the hourglass case) for a given corresponding loading frame; this is particularly visible in the third frame shown in Fig. 14c.

(a)

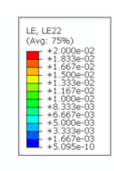

(b)
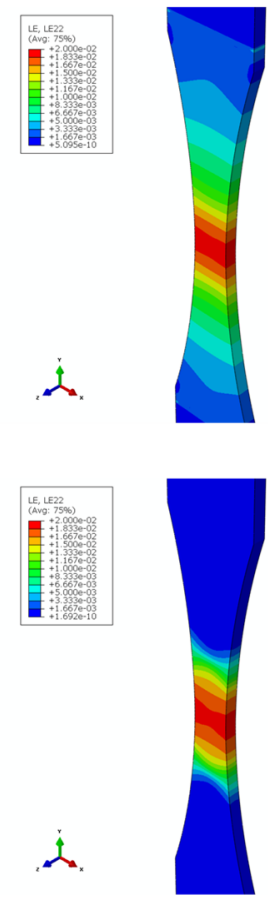
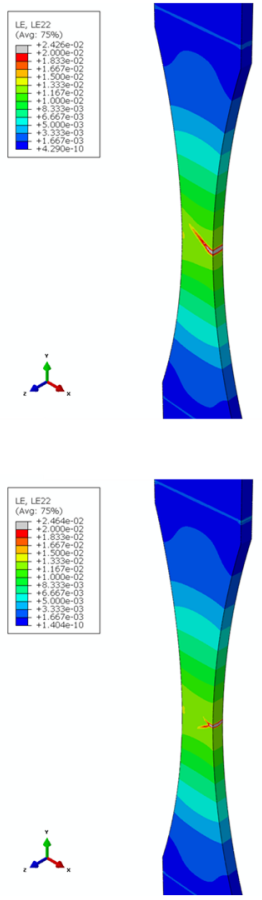

Fig. 15: Strain plots in (a) TD and (b) ND specimens with (right) or without (left) the application of the stiffness degradation step

Moreover, results from the hourglass specimen are presented in Fig. 15, both for TD and ND specimens for damaged and undamaged cases. This geometry does not introduce any imperfections (e.g. notches, width variation) to trigger a strain localization. As shown in Fig. 15a, the only way to induce such localizations is by using the plasticity law in conjunction with the proposed damage criterion. Otherwise, a homogeneous deformation is observed in all loading ranges. For the ND case in particular, one could expect that the implementation of the experimentally extracted material law would be adequate to produce localized deformation, as shown in Fig 8. However, this 
is not the case, as the material law is the average response over a specified field of view and in consequence localized effects are smeared out. A SLB is produced in this case (Fig 15b) by using a damage criterion, as described earlier.

\subsection{Load drop at the initiation of the SLB}

In order to further explore the capability of the proposed modeling approach in initiating SLBs and creating added effects similar to those observed experimentally, the results in Fig. 16 were computed. The specimen geometry chosen for this analysis is suitable to study deformation effects in a geometrically predetermined region. The overall computed stress-strain responses for both ND and TD (damaged and undamaged) specimens is shown which agree with the experimental data (given in Fig. 8). The stiffness degradation criterion used for the 'damaged' case was $\varepsilon_{c r .}^{p}=0.01$ in this example to examine the control of the criterion in initiating SLBs and form the instability after the yielding region; the parameters $d_{f}$ and $a$ were equal to 0.7 and 0.5 respectively to achieve this goal. A stress drop can be seen at $\bar{\varepsilon}_{y y} \approx 1.24 \%$ and a local minimum is reached at $\bar{\varepsilon}_{y y} \approx 1.48 \%$. Thereafter, a wavy response is obtained followed by a strain hardening behavior. Furthermore, the average longitudinal strain in the FOV as a function of the applied strain for the TD damaged and undamaged specimens is plotted in Fig. 16a. The strain in the undamaged specimen monotonically increases at an almost constant rate, while the damaged specimen follows the same path until a sudden jump at applied strain $\varepsilon \approx 0.43 \%$. This increase of strain corresponds to the range from the

stress drop to the stress local minimum, that is from $\bar{\varepsilon}_{y y} \approx 1.24 \%$ to $\bar{\varepsilon}_{y y} \approx 1.48 \%$. After this point, the strain is increasing at a higher rate. 
In Fig. 16b representative plots of the longitudinal strain field for the two cases are given.

The legend range is fixed and the time instances are marked on the stress-strain and strain evolution plots with Latin numerals and actual numbers. The top row fields reveal a necking mode for the deformation of the undamaged TD specimen. The bottom row figures depict how a SLB initiates, grows along the specimen's width and increases its thickness. It should be mentioned that no special considerations were made to model the band's thickness and growth. Consequently, the movement of the band's front was governed by the element stiffness degradation which appeared to follow the 'kinematic docking' process, described by Ramakrishnan and Atluri [6], referring to the fact that the band's fronts are nearly linear suggesting some sort of kinematic influence of elements that degrades their stiffness, as well as the stiffness of their neighbors.

(a)
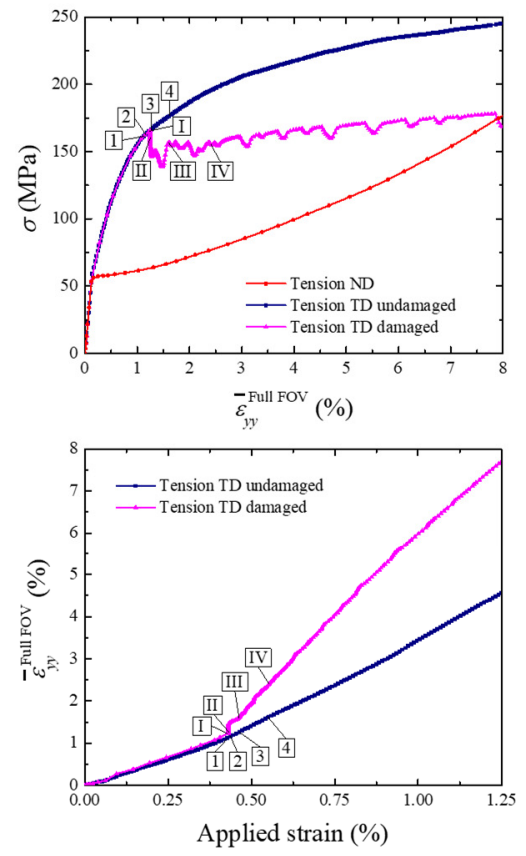

(b)
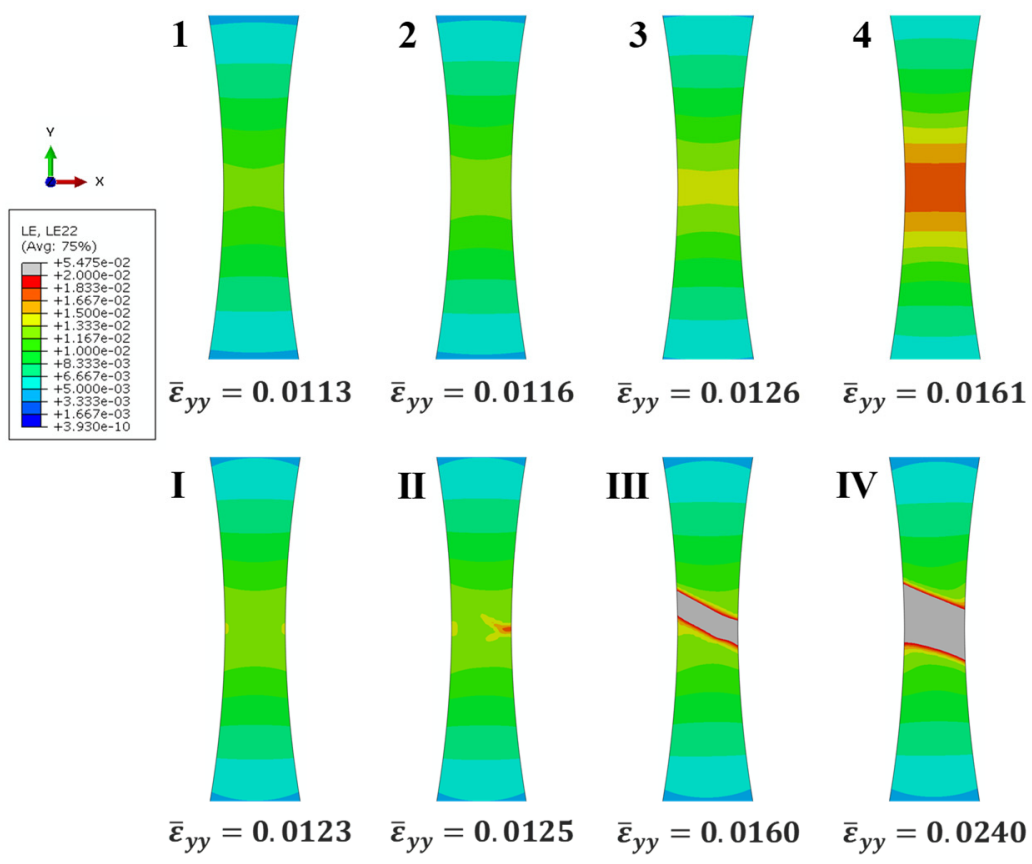

Fig. 16: (a) Stress-strain and strain evolution curves for an undamaged and a damaged TD specimens, (b) representative strain contours for the two cases. 


\subsection{Geometry of the $S L B$}

Other related geometrical properties of the computed strain localizations including their width and relative angle, were determined in Fig. 17. The range was fixed while various mesh densities were employed to investigate the repeatability of these calculations. It should be mentioned at this point, that these numerical results were calculated by averaging values at nodes common to two or more elements when these elements lied in the same region. A threshold was applied to control such averaging and smooth the solution. Under these conditions, the results for the strain localization width and relative angle evolution are presented in Fig. 17.

As mentioned earlier, the SLB starts forming at applied strain $\varepsilon \approx 0.43 \%$. After growing through the specimen's width, the band's fronts are moving at almost a constant rate as indicated by the linear trend shown in Fig. 17a. It should be reminded that the mesh dependence study presented earlier suggested a mesh density of $0.6 \mathrm{~mm}$ for convergent solutions. Accordingly, the shear band angle evolution as a function of applied strain is shown in Fig. 17b. As the band forms and its thickness starts to grow, the angle increases up to a maximum value of $\sim 32.5^{\circ}$, which is in agreement with the analytical prediction of $\theta=\nu^{1 / 2} \approx 33^{\circ}$, where $\nu$ is the Poisson ratio [36]. The angle is then affected by the specimen's geometry and the accumulated strain in the SLB and therefore decreases as the band approaches the wider parts of the specimen. 
(a)

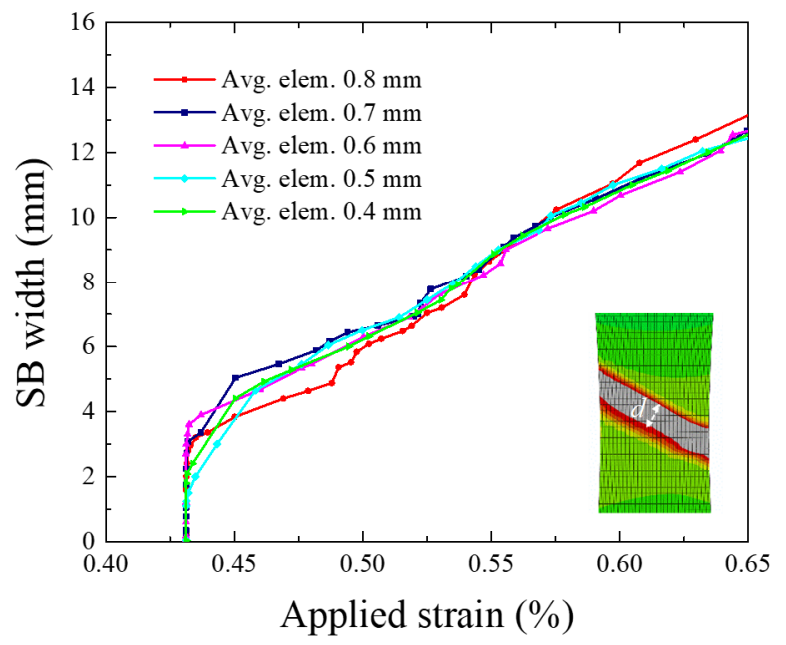

(b)

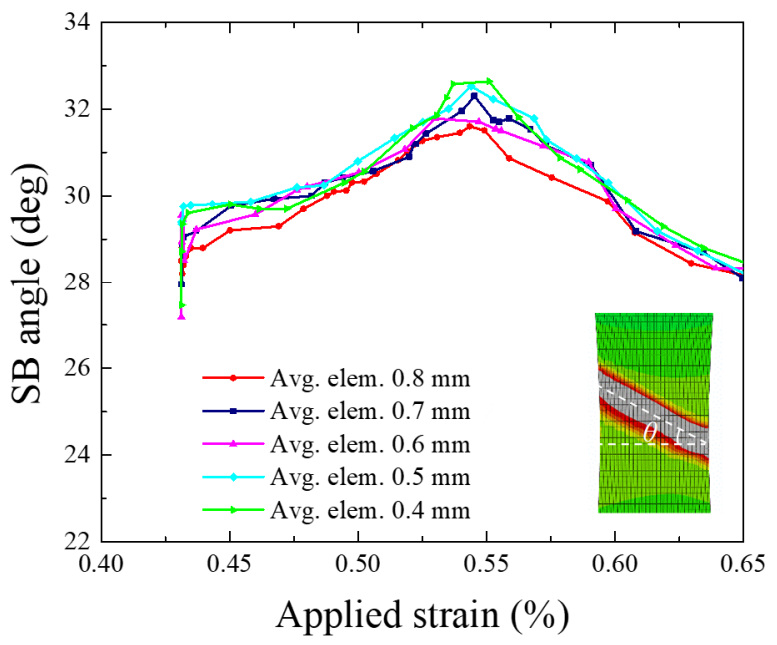

Fig. 17: SLB (a) thickness and (b) angle as a function of applied strain for different mesh sizes

\section{Concluding remarks}

The effects of strain localizations in the form of bands in magnesium alloys were investigated in this article through an integrated experimental and computational approach. The experimental portion of this investigation targeted the comparison of the local and bulk mechanical behavior of specimens that had predetermined textures that either favored or not the activation of deformation twinning. The specific geometry of the specimens used facilitated the comparison of the strain localizations that were induced by the externally applied tensile load, while further allowing post mortem microscopic investigation. Overall, three measurable effects were captured. The first relates to the strain accumulation inside such bands, the second on the initiation of band formation and the third on the softening observed past this initiation. Microscopic investigation showed twin activity near the strain localization band fronts. In addition, the strains measured within the localization regions were found to be significantly larger in the specimens which were favorable to twinning. 
Given these experimental inputs, bands in the computational approach were not triggered by any artificial change in geometry (e.g. side indentation, thickness alteration) but were instigated through a stiffness degradation step, in accordance with the softening that was measured. Furthermore, the simulation results agreed well with the corresponding experimental data demonstrating that strain localization band formation affects both local, as manifested by the strain accumulation in the band regions, and bulk mechanical behavior as computed by effects observed at the stress-strain level. On the other hand, the presented integrated method does not eliminate the mesh dependence often observed in strain localization simulations. In conclusion, this investigation contributes in the understanding of the role of strain localization bands on the mechanical behavior of magnesium allows, while it also allows for further refinement of the constitutive description of such instabilities.

\section{Acknowledgments}

The corresponding author acknowledges the financial support provided by the National Science Foundation through the CMMI \#1434506 award to Drexel University. He also acknowledges the technical support received under the National Aeronautics and Space Administration Space Act Agreement, No. SAA1-19439 with Langley Research Center. The results reported were obtained by using computational resources supported by Drexel's University Research Computing Facility. This investigation was also supported by the funds received in terms of fellowship to M. Cabal from the Greater Philadelphia Region Louis Strokes Alliance for Minority Participation. 


\section{Appendix}

\section{Material}

Two sets of samples were machined from two different commercial magnesium AZ31 alloy (3 wt. \% Al and 1 wt. \% Zn) plates. The notched specimens were prepared from a $25.4 \mathrm{~mm}$ thick plate in the soft annealed condition (O temper). Two cubes were extruded from the plate and then compressed to $5 \%$ load in either the normal to rolling (ND) or transverse to rolling (TD) direction. The cubes were later held at $500^{\circ} \mathrm{C}$ for 50 hours to increase their grain size. Texture and grain size were measured in samples cut from the normal direction. The straight specimens were prepared from a $50.8 \mathrm{~mm}$ thick plate in $\mathrm{H} 24$ condition. Cubes were cut from the plate and heat treated to O temper at $350^{\circ} \mathrm{C}$ for 1 hour. Both tensile specimens were machined from the cubes using Electrical Discharged Machining (EDM) according to ASTM E606.

Samples were mechanically ground using 800, 1200, and $2400 \mathrm{SiC}$ papers. Subsequent polishing was carried out using an alcohol based diamond solution through a sequence of $6 \mu \mathrm{m}, 3 \mu \mathrm{m}$, and $1 \mu \mathrm{m}$ Buehler's TriDent and Struers' Nap polishing cloths. Polishing was completed using a

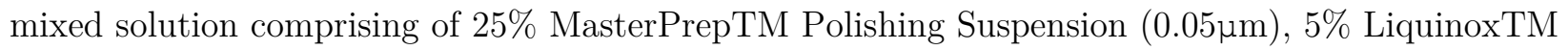
critical-cleaning liquid detergent, and $70 \%$ water. In each polishing step, ultrasonic cleansing was used to completely eliminate adhered particles from previous polishing courses. Specimens were then immersed in a chemical polishing solution comprised of 5\% nitric acid, $15 \%$ acetic acid, 20\% distilled water, and $60 \%$ ethanol for 3 seconds.

All EBSD data was collected with a Scanning Electron Microscope (SEM) FEI XL30 equipped with an EBSD detector, controlled by the TSL software. Grain information, including 
lattice orientations and grain size, were measured at intervals of $2 \mu \mathrm{m}$ on a hexagonal grid by automated acquisition and processing of backscatter diffraction patterns. The accelerating voltage and working distance were set at $30 \mathrm{kV}$ and $15 \mathrm{~mm}$, respectively. EBSD data was post processed using MTEX algorithm in MATLAB platform [37].

\section{Mechanical Testing}

A speckle pattern was applied on the surface of samples before testing for DIC measurements. A white paint coating was sprayed using at aerosol canister, while black dots were scattered on top using an airbrush. The notched samples were tested using a MTS 858 table top system with a load cell of 3 kips $(13.35 \mathrm{kN})$. The testing was carried out using displacement control, at a rate of $5.315 \times 10^{-4} \mathrm{in} / \mathrm{s}\left(1.35 \times 10^{-2} \mathrm{~mm} / \mathrm{s}\right)$. Experiments of the ND and TD specimens both to failure and to a pre-determined value of engineering strain are reported in this article. DIC was carried out by an optical microscope with dual (2) cameras in concurrence with the commercial software VIC-3D (commercially available by Correlated Solutions, Inc.) monitored deformation in a Field of View (FOV) of $5 \mathrm{~mm} \times 6 \mathrm{~mm}$ size during two tension tests (Fig. 1). Along with the selected FOV, a narrower diagonal area marked in Fig. 1 was used to quantify the deformation of the shear band region only.

The straight specimens were tested with a screw-driven Gatan MTEST stage with a $2000 \mathrm{~N}$ load cell. The tests were conducted using displacement control with a rate of $0.1 \mathrm{~mm} / \mathrm{min}$. The Gatan stage has pre-tilted grips providing the capability for in-situ EBSD measurement testing inside of SEM. DIC measurements were attained using a GOM (Trillion) 5M dual cameras setup and the ARAMIS software. 


\section{References}

1. Pietruszczak S, Mróz Z (1981) Finite element analysis of deformation of strain-softening materials. International Journal for Numerical Methods in Engineering 17 (3):327-334. doi:10.1002/nme.1620170303

2. Tvergaard V, Needleman A, Lo KK (1981) Flow localization in the plane strain tensile test. Journal of the Mechanics and Physics of Solids 29 (2):115-142. doi:http://dx.doi.org/10.1016/0022$\underline{5096(81) 90019-3}$

3. Ortiz M, Leroy Y, Needleman A (1987) A finite element method for localized failure analysis. Computer Methods in Applied Mechanics and Engineering 61 (2):189-214. doi:http://dx.doi.org/10.1016/0045-7825(87)90004-1

4. Belytschko T, Fish J, Engelmann BE (1988) A finite element with embedded localization zones. Computer Methods in Applied Mechanics and Engineering $70 \quad$ (1):59-89. doi:http://dx.doi.org/10.1016/0045-7825(88)90180-6

5. Batra RC, Kim CH (1992) Analysis of shear banding in twelve materials. International Journal of Plasticity 8 (4):425-452. doi:http://dx.doi.org/10.1016/0749-6419(92)90058-K

6. Ramakrishnan N, Atluri SN (1994) Simulation of shear band formation in plane strain tension and compression using FEM. Mechanics of Materials 17 (2-3):307-317. doi:http://dx.doi.org/10.1016/0167-6636(94)90068-X

7. Shaw JA, Kyriakides S (1997) Initiation and propagation of localized deformation in elasto-plastic strips under uniaxial tension. International Journal of Plasticity 13 (10):837-871. doi:http://dx.doi.org/10.1016/S0749-6419(97)00062-4

8. Tsukahara H, Iung T (1998) Finite element simulation of the Piobert-Lüders behavior in an uniaxial tensile test. Materials Science and Engineering: A 248 (1-2):304-308. doi:http://dx.doi.org/10.1016/S0921-5093(97)00857-5

9. Koplik J, Needleman A (1988) Void growth and coalescence in porous plastic solids. International Journal of Solids and Structures 24 (8):835-853. doi:http://dx.doi.org/10.1016/0020-7683(88)90051$\underline{0}$ 
10. Kuroda M, Tvergaard V (2007) Effects of texture on shear band formation in plane strain tension/compression and bending. International Journal of Plasticity 23 (2):244-272. doi:http://dx.doi.org/10.1016/j.ijplas.2006.03.014

11. Jia N, Roters F, Eisenlohr P, Kords C, Raabe D (2012) Non-crystallographic shear banding in crystal plasticity FEM simulations: Example of texture evolution in $\alpha$-brass. Acta Materialia 60 (3):1099-1115. doi:http://dx.doi.org/10.1016/j.actamat.2011.10.047

12. Jia N, Eisenlohr P, Roters F, Raabe D, Zhao X (2012) Orientation dependence of shear banding in face-centered-cubic single crystals. Acta Materialia 60 (8):3415-3434. doi:http://dx.doi.org/10.1016/j.actamat.2012.03.005

13. Paul H, Morawiec A, Driver JH, Bouzy E (2009) On twinning and shear banding in a Cu-8 at.\% $\mathrm{Al}$ alloy plane strain compressed at $77 \mathrm{~K}$. International Journal of Plasticity 25 (8):1588-1608. doi:http://dx.doi.org/10.1016/j.ijplas.2008.10.003

14. Hazeli K, Cuadra J, Vanniamparambil PA, Kontsos A (2013) In situ identification of twinrelated bands near yielding in a magnesium alloy. Scripta Materialia 68 (1):83-86. doi:http://dx.doi.org/10.1016/j.scriptamat.2012.09.009

15. Aydiner CC, Telemez MA (2014) Multiscale deformation heterogeneity in twinning magnesium investigated with in situ image correlation. International Journal of Plasticity 56:203-218. doi:http://dx.doi.org/10.1016/j.ijplas.2013.12.001

16. Hazeli K, Cuadra J, Streller F, Barr CM, Taheri ML, Carpick RW, Kontsos A (2015) Threedimensional effects of twinning in magnesium alloys. Scripta Materialia 100:9-12. doi:http://dx.doi.org/10.1016/j.scriptamat.2014.12.001

17. Barnett MR, Nave MD, Ghaderi A (2012) Yield point elongation due to twinning in a magnesium alloy. Acta Materialia 60 (4):1433-1443. doi:http://dx.doi.org/10.1016/j.actamat.2011.11.022 18. Changizian P, Zarei-Hanzaki A, Ghambari M, Imandoust A (2013) Flow localization during severe plastic deformation of AZ81 magnesium alloy: Micro-shear banding phenomenon. Materials Science and Engineering: A 582:8-14. doi:http://dx.doi.org/10.1016/j.msea.2013.05.069 
19. Changizian P, Zarei-Hanzaki A, Abedi HR (2012) On the recrystallization behavior of homogenized AZ81 magnesium alloy: The effect of mechanical twins and $\curlyvee$ precipitates. Materials Science and Engineering: A 558:44-51. doi:http://dx.doi.org/10.1016/j.msea.2012.07.054

20. Chun YB, Davies CHJ (2011) Texture effect on microyielding of wrought magnesium alloy $\begin{array}{llllll}\text { AZ31. Materials } & \text { Science and } & \text { Engineering: } & \text { A } & 528 & \text { (9) }\end{array}$ doi:http://dx.doi.org/10.1016/j.msea.2011.01.046

21. Fatemi-Varzaneh SM, Zarei-Hanzaki A, Cabrera JM (2011) Shear banding phenomenon during severe plastic deformation of an AZ31 magnesium alloy. Journal of Alloys and Compounds 509 (9):3806-3810. doi:http://dx.doi.org/10.1016/j.jallcom.2011.01.019

22. Li X, Yang P, Wang LN, Meng L, Cui F (2009) Orientational analysis of static recrystallization at compression twins in a magnesium alloy AZ31. Materials Science and Engineering: A 517 (12):160-169. doi:http://dx.doi.org/10.1016/j.msea.2009.03.045

23. ABAQUS (2013). version 6.13, 2013. User's Manual. Dassault Systems, Pawtucket, RI.

24. Cazacu O, Plunkett B, Barlat F (2006) Orthotropic yield criterion for hexagonal closed packed

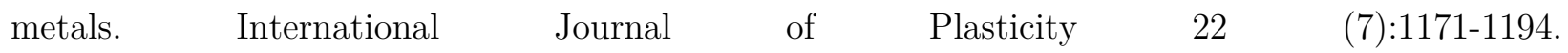
doi:http://dx.doi.org/10.1016/j.ijplas.2005.06.001

25. Lee M-G, Wagoner RH, Lee JK, Chung K, Kim HY (2008) Constitutive modeling for anisotropic/asymmetric hardening behavior of magnesium alloy sheets. International Journal of Plasticity 24 (4):545-582. doi:http://dx.doi.org/10.1016/j.ijplas.2007.05.004

26. Ramaswamy S, Aravas N (1998) Finite element implementation of gradient plasticity models Part II: Gradient-dependent evolution equations. Computer Methods in Applied Mechanics and Engineering 163 (1-4):33-53. doi:http://dx.doi.org/10.1016/S0045-7825(98)00027-9

27. de Borst R, L.J. Sluys LJ, Mühlhaus H-B, Pamin J (1993) Fundamental issues in finite element analyses of localization of deformation. Engineering Computations 10 (2):99-121. doi:doi:10.1108/eb023897

28. Needleman A (1988) Material rate dependence and mesh sensitivity in localization problems. Computer Methods in Applied Mechanics and Engineering 67 (1):69-85. doi:http://dx.doi.org/10.1016/0045-7825(88)90069-2 
29. Niezgoda SR, Kanjarla AK, Beyerlein IJ, Tomé CN (2014) Stochastic modeling of twin nucleation in polycrystals: An application in hexagonal close-packed metals. International Journal of Plasticity 56:119-138. doi:http://dx.doi.org/10.1016/j.ijplas.2013.11.005

30. Yoo MH (1981) Slip, twinning, and fracture in hexagonal close-packed metals. Metallurgical Transactions A 12 (3):409-418. doi:10.1007/bf02648537

31. Beyerlein IJ, Capolungo L, Marshall PE, McCabe RJ, Tomé CN (2010) Statistical analyses of deformation twinning in magnesium. Philosophical Magazine 90 (16):2161-2190. doi:10.1080/14786431003630835

32. Yu Q, Jiang Y, Wang J (2015) Cyclic deformation and fatigue damage in single-crystal magnesium under fully reversed strain-controlled tension-compression in the $\left[\begin{array}{lll}1 & 0 & 0\end{array}\right]$ direction. Scripta Materialia 96:41-44. doi:http://dx.doi.org/10.1016/j.scriptamat.2014.10.020

33. Mo C, Wisner B, Cabal M, Hazeli K, Ramesh K, El Kadiri H, Al-Samman T, Molodov K, Molodov D, Kontsos A (2016) Acoustic Emission of Deformation Twinning in Magnesium. Materials $9(8): 662$

34. Liu X, Jonas JJ, Li LX, Zhu BW (2013) Flow softening, twinning and dynamic recrystallization in AZ31 magnesium. Materials Science and Engineering: A 583:242-253. doi:http://dx.doi.org/10.1016/j.msea.2013.06.074

35. Sun HB, Yoshida F, Ma X, Kamei T, Ohmori M (2003) Finite element simulation on the propagation of Lüders band and effect of stress concentration. Materials Letters 57 (21):3206-3210. doi:http://dx.doi.org/10.1016/S0167-577X(03)00036-3

36. Oliver J (1996) Modelling strong discontinuities in solid mechanics vis strain softening constitutive equations. Part 1: Fundamentals. International Journal for Numerical Methods in Engineering $39 \quad$ (21):3575-3600. doi:10.1002/(SICI)1097-0207(19961115)39:21<3575::AIDNME65 > 3.0.CO;2-E

37. Hielscher R, Schaeben H (2008) A novel pole figure inversion method: specification of the MTEX $\begin{array}{llllll}\text { algorithm. Journal of } & \text { Applied } & \text { Crystallography } & 41024-1037 .\end{array}$ doi:doi:10.1107/S0021889808030112 